Taira, $\mathrm{K}$.

Osaka J. Math.

33 (1996), 555-585

\title{
ON BOUNDARY VALUE PROBLEMS OF NONLINEAR ELASTOSTATICS
}

Dedicated to the memory of Professor Hitoshi Kumano-go

\author{
KAZUAKI TAIRA
}

(Received March 3, 1994)

\section{Introduction and results}

This paper is devoted the $L^{p}$ approach to genuine mixed displacement-traction boundary value problems of nonlinear elastostatics. Our boundary condition is a "regularization" of the genuine mixed displacement-traction boundary condition; more precisely, it is a smooth linear combination of displacement and traction boundary conditions, but is not equal to the pure traction boundary condition. The crucial point is how to find a function space associated with the boundary condition in which the linearized problem has a unique solution. Our result can be applied to the St. Venant-Kirchhoff elastic material and the Hencky-Nadai elasto-plastic material. Some previous results with pure displacement boundary condtion are due to Ciarlet [4], Dinca [5], Marsden-Hughes [11] and Valent [16]. The results here extend and improve substantially those results in a unified theory.

Let $\Omega$ be an open, connected subset of Euclidean space $\boldsymbol{R}^{3}$ with piecewise smooth boundary $\partial \Omega$. We think of the closure $\bar{\Omega}=\Omega \cup \partial \Omega$ as representing the volume occupied by an undeformed body; so the set $\mathscr{B}=\bar{\Omega}$ is called the reference configuration. A configuration of $\mathscr{B}$ is a $C^{1}$ map $\phi: \mathscr{B} \rightarrow \boldsymbol{R}^{3}$ which is orientation-preserving and invertible. A configuration represents a deformed state of the body. Points in $\mathscr{B}$ are denoted by $X=\left(X_{1}, X_{2}, X_{3}\right)$ and are called material points, while points in $\boldsymbol{R}^{3}$ are denoted by $x=\left(x_{1}, x_{2}, x_{3}\right)$ and are called spatial points. We write as $x=\phi(X)$.

The $3 \times 3$ matrix of partial derivatives of $\phi$ is denoted by $F(X)=D \phi(X)$ and is called the deformation gradient. The symmetric tensor $\boldsymbol{C}(\boldsymbol{F})={ }^{t} \boldsymbol{F F}$ is called the Green deformation tensor.

A body $\phi(\mathscr{B})$ is acted on by applied body forces $b(x)$ in its interior and by applied surface forces $\tau(x)$ on a portion of the boundary. The pair $(\boldsymbol{b}, \tau)$ of forces is called the load.

In additon, the body generally experiences internal forces of stress across any given surface. Let $t(x, n)$ be the force at position $x$ across an oriented surface element with outward unit normal $n$. The celebrated Cauchy theorem asserts that if the balance of momentum holds, then the stress vector $t(x, n)$ depends 
linearly on $n$, that is, there exists a symmetric two-tensor $\sigma(x)$ such that

$$
\boldsymbol{t}(x, \boldsymbol{n})=\sigma(x) \cdot n ; \quad t_{i}(x, \boldsymbol{n})=\sum_{j=1}^{3} \sigma_{i j}(x) n_{j} .
$$

The vector $t(x, n)$ is called the Cauchy stress vector and the tensor $\sigma(x)$ is called the Cauchy stress tensor.

The vector $T(X, N)$, defined by the formula

$$
T(X, N)=\boldsymbol{P}(X) \cdot N(X), \quad \boldsymbol{P}(X)=\operatorname{det}(D \phi(X)) \boldsymbol{\sigma}(\phi(X)) \cdot\left({ }^{t} D \phi(X)\right)^{-1},
$$

is called the first Piola-Kirchhoff stress vector, where $N(X)$ is the outward unit normal to the boundary $\partial \Omega$ at $X$. The two-tensor $\boldsymbol{P}(X)$, which is the Piola transform of the Cauchy stress tensor $\sigma(x)$, is called the first Piola-Kirchhoff stress tensor.

A material is said to be elastic if one can write the first Piola-Kirchhoff stress tensor $\boldsymbol{P}(X)$ as a function $\hat{\boldsymbol{P}}(X, \boldsymbol{F})$ of points $X \in \mathscr{B}$ and $3 \times 3$ matrices $\boldsymbol{F}=\left(F_{i j}\right)$ with $\operatorname{det} F>0$ such that

$$
\boldsymbol{P}(X)=\hat{\boldsymbol{P}}(X, D \phi(X)) \text {. }
$$

An elastic material is said to be hyperelastic if there exists a smooth function $W(X, F)$ of points $X \in \mathscr{B}$ and $3 \times 3$ matrices $\boldsymbol{F}$ with $\operatorname{det} \boldsymbol{F}>0$ such that

$$
\hat{\boldsymbol{P}}(X, \boldsymbol{F})=\frac{\partial W}{\partial \boldsymbol{F}}(X, \boldsymbol{F}) ; \quad \hat{P}_{i j}(X, \boldsymbol{F})=\frac{\partial W}{\partial F_{i j}}(X, \boldsymbol{F}) .
$$

The function $W(X, F)$ is called a stored energy function. The four-index tensor $\mathbf{A}=\partial \hat{\boldsymbol{P}} / \partial \boldsymbol{F}=\partial^{2} W / \partial \boldsymbol{F} \partial \boldsymbol{F}$, defined by the formula

$$
\mathrm{A}_{i j l m}(X, \boldsymbol{F})=\frac{\partial \hat{P}_{i j}}{\partial F_{l m}}(X, \boldsymbol{F})=\frac{\partial^{2} W}{\partial F_{i j} \partial F_{l m}}(X, \boldsymbol{F}),
$$

is called the first elasticity tensor.

We make the following two assumptions throughout the paper:

(H.1) The reference configuration is a bounded region $\mathscr{B}=\bar{\Omega} \subset R^{3}$ with smooth boundary $\partial \Omega$.

(H.2) The material is hyperelastic.

Some boundary conditions often encountered are the following:

(a) Displacement $\phi(X)=\phi_{d}(X)$ is described for $X \in \partial \Omega$.

(b) Traction $\hat{\boldsymbol{P}}(X, D \phi(X)) \cdot \boldsymbol{N}(X)=\boldsymbol{\tau}(X)$ is described for $X \in \partial \Omega$.

Now let $B: \Omega \rightarrow R^{3}$ be the density of a given body force per unit volume in the reference configuration and $\tau: \partial \Omega \rightarrow \boldsymbol{R}^{3}$ the density of a given surface force per unit area in the reference configuration. In this paper we consider the following equilibrium equations for the unknown configuration $\phi$ : 
(*) $\begin{cases}\operatorname{div} \hat{\boldsymbol{P}}(X, D \phi(X))+\boldsymbol{B}(X)=\mathbf{0} & \text { in } \Omega, \\ \alpha(X) \hat{\boldsymbol{P}}(X, D \phi(X)) \cdot N(X)+(1-\alpha(X)) \phi(X)=\tau(X) & \text { on } \partial \Omega,\end{cases}$

where $\alpha$ is a smooth funciton on $\partial \Omega$ such that

$$
0 \leq \alpha \leq 1 \quad \text { on } \partial \Omega \text {. }
$$

We remark that our problem $(*)$ is a "regularized" genuine mixed displacementtraction boundary value problem.

We study the nonlinear problem $(*)$ in the framework of Sobolev spaces of $L^{p}$ style. If $s \in \boldsymbol{R}$ and $1<p<\infty$, we define the Sobolev space (see Subsection 1.1)

$$
H^{s, p}(\Omega)=\text { the space of restrictions to } \Omega \text { of functions in } H^{s, p}\left(\boldsymbol{R}^{n}\right) \text {. }
$$

The space $H^{s, p}(\Omega)$ is a Banach space with the norm

$$
\|u\|_{s, p}=\inf \left\{\|U\|_{s, p} ; U \in H^{s, p}\left(R^{n}\right),\left.U\right|_{\Omega}=u\right\} .
$$

Furthermore, if $s>1 / p$, we define

$$
\begin{aligned}
B^{s-1 / p, p}(\partial \Omega)= & \text { the space of the boundary values } \varphi \text { of functions } \\
& u \in H^{s, p}(\Omega)
\end{aligned}
$$

with the norm

$$
|\varphi|_{s-1 / p, p}=\inf \left\{\|u\|_{s, p} ; u \in H^{s, p}(\Omega),\left.u\right|_{\partial \Omega}=\varphi\right\} .
$$

The space $B^{s-1 / p, p}(\partial \Omega)$ is a Banach space with respect to the norm $|\cdot|_{s-1 / p, p}$; more precisely it is a Besov space.

We let

$$
\begin{aligned}
& \boldsymbol{H}^{s, p}\left(\Omega, \boldsymbol{R}^{3}\right)=\text { the space of all } H^{s, p} \text { functions } \phi: \Omega \rightarrow \boldsymbol{R}^{3}, \\
& \boldsymbol{B}^{s-1 / p, p}\left(\partial \Omega, \boldsymbol{R}^{3}\right)=\text { the space of all } B^{s-1 / p, p} \text { functions } \varphi: \partial \Omega \rightarrow \boldsymbol{R}^{3} .
\end{aligned}
$$

We introduce a subspace of the Besov space $B^{s-1-1 / p, p}\left(\partial \Omega, R^{3}\right)$ for $s>1+1 / p$ which is associated with the boundary condition

$$
\alpha \hat{P}(D \phi) \cdot N+(1-\alpha) \phi=\tau \quad \text { on } \partial \Omega
$$

in the following way: We let

$$
\begin{gathered}
B_{(\alpha)}^{s-1-1 / p, p}\left(\partial \Omega, R^{3}\right)=\left\{\varphi=\alpha \varphi_{1}+(1-\alpha) \varphi_{2} ; \varphi_{1} \in B^{s-1-1 / p, p}\left(\partial \Omega, R^{3}\right),\right. \\
\left.\varphi_{2} \in B^{s-1 / p, p}\left(\partial \Omega, R^{3}\right)\right\}
\end{gathered}
$$

with the norm 


$$
|\varphi|_{\alpha ; s-1-1 / p, p}=\inf \left\{\left|\varphi_{1}\right|_{s-1-1 / p, p}+\left|\varphi_{2}\right|_{s-1 / p, p} ; \varphi=\alpha \varphi_{1}+(1-\alpha) \varphi_{2}\right\} .
$$

Then it is easy to verify that the space $B_{(\alpha)}^{s-1-1 / p, p}\left(\partial \Omega, R^{3}\right)$ is a Banach space with respect to the norm $|\cdot|_{\alpha ; s-1-1 / p, p}$. We remark that

$$
\begin{aligned}
& \boldsymbol{B}_{(\alpha)}^{s-1-1 / p, p}\left(\partial \Omega, \boldsymbol{R}^{3}\right)=B^{s-1 / p, p}\left(\partial \Omega, \boldsymbol{R}^{3}\right) \quad \text { if } \alpha \equiv 0 \text { on } \partial \Omega \text { (displacement), } \\
& \boldsymbol{B}_{(\alpha)}^{s-1-1 / p, p}\left(\partial \Omega, \boldsymbol{R}^{3}\right)=\boldsymbol{B}^{s-1-1 / p, p}\left(\partial \Omega, \boldsymbol{R}^{3}\right) \quad \text { if } \alpha \equiv 1 \text { on } \partial \Omega \text { (traction). }
\end{aligned}
$$

If $s>3 / p+1$, we let

$$
\mathscr{C}=\text { the subspace of all configurations } \phi \text { in } \boldsymbol{H}^{s, p}\left(\Omega, R^{3}\right) \text {. }
$$

We remark that the set $\mathscr{C}$ is open in the space $H^{s, p}\left(\Omega, R^{3}\right)$. Indeed, this follows from an application of the inverse mapping theorem, since the Sobolev imbedding theorem tells us that the $H^{s, p}$ topology is stronger than the $C^{1}$ topology, for all $s>3 / p+1$.

We associate with problem $(*)$ a nonlinear map between Banach spaces

$$
F: H^{s, p}\left(\Omega, R^{3}\right) \rightarrow H^{s-2, p}\left(\Omega, R^{3}\right) \times B_{(\alpha)}^{s-1-1 / p, p}\left(\partial \Omega, R^{3}\right)
$$

as follows:

$$
F(\phi)=\left(-\operatorname{div} \hat{\boldsymbol{P}}(D \phi), \alpha \hat{\boldsymbol{P}}(D \phi) \cdot \boldsymbol{N}+\left.(1-\alpha) \phi\right|_{\partial \Omega}\right), \quad \phi \in \mathscr{C} .
$$

It follows from an application of the $\omega$-lemma (see [11, Chapter 3, Theorem 1.13], [16, Chapter II, Section 4]) that the map $F$ is of class $C^{1}$.

Now we can state our main existence and uniqueness result for problem (*) of nonlinear elastostatics:

Main theorem. Let $1<p<\infty$ and $s>3 / p+1$. We assume that:

(P) $\hat{\boldsymbol{P}}(D \stackrel{\phi}{)})=\mathbf{0}$ when $\stackrel{\boldsymbol{\phi}}{=} \boldsymbol{I}_{\boldsymbol{\Omega}}$ (identity map on $\Omega$ ).

(H) The first elasticity tensor $\AA=\partial^{2} W / \partial \boldsymbol{F} \partial \boldsymbol{F}$ evaluated at $\stackrel{\phi}{\phi}=\boldsymbol{I}_{\Omega}$ enjoys the property of symmetry

$$
\AA_{i j l m}=\AA_{j i l m}\left(=\AA_{l m i j}\right),
$$

and is uniformly pointwise stable, that is, there is a constant $\eta>0$ such that

$$
\frac{1}{2} \boldsymbol{e} \cdot \AA \cdot e \geq \eta\|\boldsymbol{e}\|^{2}
$$

for all symmetric two tensors $\boldsymbol{e}$.

(A) $\alpha \not \equiv 1$ on $\partial \Omega$.

Then there exist a neighborhood $\mathscr{U}$ of the configuration $\$$ in $H^{s, p}\left(\Omega, R^{3}\right)$ and a neighborhood $\mathscr{V}$ of the point 


$$
\left(-\operatorname{div} \hat{\boldsymbol{P}}(D \AA), \alpha \hat{\boldsymbol{P}}(D \AA) \cdot N+\left.(1-\alpha) \dot{\phi}\right|_{\partial \Omega}\right)
$$

in $\boldsymbol{H}^{s-2, p}\left(\Omega, \boldsymbol{R}^{3}\right) \times \boldsymbol{B}_{(\alpha)}^{s-1-1 / p, p}\left(\partial \Omega, \boldsymbol{R}^{3}\right)$ such that the map $F: \mathscr{U} \rightarrow \mathscr{V}$ is one-to-one and onto.

Condition (P) implies that the undeformed state is stress free. Condition (A) implies that our boundary condition is not equal to the pure traction boundary condition. It is worth pointing out here that the pure traction problem may have non-unique solutions even for small loads and near a stress free state (see [11, Chapter 7, Section 7.3]).

Rephrased, Main Theorem states that if the linearized problem is uniformly pointwise stable, then, for slight perturbations of the load or boundary conditions from their values at the undeformed state, the nonlinear problem $(*)$ has a unique solution $\phi$ near $\stackrel{\circ}{\phi}=I_{\Omega}$.

We give two examples of hyperelastic materials.

EXAMPLE 1 (The Hencky-Nadai elasto-plastic material). The stored energy function $W(X, \boldsymbol{F})$ has the form

$$
W(X, F)=\frac{3}{4} \int_{0}^{T(\boldsymbol{F})} g(\xi) d \xi+\frac{K}{2}\left(\sum_{k=1}^{3} F_{k k}-3\right)^{2},
$$

where $g \in C^{\infty}([0, \infty), R), K$ is the modulus of compression and

$$
\Gamma(F)=\frac{4}{3} \sum_{i, j=1}^{3}\left(\frac{1}{2}\left(F_{i j}+F_{j i}\right)-\frac{1}{3}\left(\sum_{k=1}^{3} F_{k k} F_{k k}\right) \delta_{i j}\right)^{2} .
$$

EXAMPLE 2 (The St.Venant-Kirchhoff isotropic material). The stored energy function $W(X, \boldsymbol{F})$ has the form

$$
W(X, F)=\frac{\lambda(X)}{8}\left(\sum_{k=1}^{3} C_{k k}(F)-3\right)^{2}+\frac{\mu(X)}{4} \sum_{i, j=1}^{3}\left(C_{i j}(F)-\delta_{i j}\right)^{2},
$$

where $\lambda(X), \mu(X)$ are smooth functions on $\bar{\Omega}$ and $C_{i j}(F)=\sum_{k=1}^{3} F_{k i} F_{k j}$ is the Green deformation tensor.

For the Hencky-Nadai elasto-plastic material, we have the following result (cf. [5, Théorème 2]):

Theorem 1. Let $1<p<\infty$ and $s>3 / p+1$. We assume that:

(A) $\alpha \neq 1$ on $\partial \Omega$.

(G) $g(0)>0$ and $K>0$.

Then condition $(H)$ is satisfied and so Main Theorem applies.

For the St.Venant-Kirchhoff isotropic material, we have the following result (cf. 
[4, Theorem 6.7-1]):

Theorem 2. Let $1<p<\infty$ and $s>3 / p+1$. We assume that:

(A) $\alpha \neq \equiv 1$ on $\partial \Omega$.

(M) There exist constants $c_{1}>0$ and $c_{2}>0$ such that

$$
\mu(X) \geq c_{1}, \lambda(X)+\frac{2}{3} \mu(X) \geq c_{2} \quad \text { on } \bar{\Omega} .
$$

Then condition $(\mathrm{H})$ is satisfied and so Main Theorem applies.

The rest of this paper is organized as follows.

In Section 1 we present a brief description of the basic concepts and results of the $L^{p}$ theory of pseudo-differential operators.

In Section 2 we linearize problem $(*)$ and study the following problem of linear elastostatics for the unknown vector function $v$ :

$$
\begin{cases}A v:=\operatorname{div}(\mathbf{a} \cdot \nabla v)=f & \text { in } \Omega, \\ B_{\alpha} v:=\alpha(\mathbf{a} \cdot \nabla v \cdot n)+(1-\alpha) v=\varphi & \text { on } \partial \Omega .\end{cases}
$$

Here a is smooth elasticity tensor and $\boldsymbol{n}$ is the outward unit normal to $\partial \Omega$.

In Sections 3 through 6 we study the linearized problem $(\dagger)$ in the framework of Sobolev spaces of $L^{p}$ style, by using the $L^{p}$ theory of pseudo-differential operators. Our fundamental existence and uniqueness theorem for problem ( $\dagger$ ) is stated as Theorem 2.1 in Section 2.

In Section 3 we show that problem $(\dagger)$ can be reduced to the study of a $3 \times 3$ matrix-valued pseudo-differential operator on the boundary. We explain more precisely the idea of our approach to problem $(\dagger)$.

First we consider the displacement boundary value problem

$$
\begin{cases}A v=\operatorname{div}(\mathbf{a} \cdot \nabla v)=f & \text { in } \Omega, \\ v=\varphi & \text { on } \partial \Omega\end{cases}
$$

The existence and uniqueness theorem for problem (D) is well established in the framework of Sobolev spaces of $L^{p}$ style (Theorem 3.1). Thus one can introduce the Possion operator

$$
\mathscr{P}: \boldsymbol{B}^{s-1 / p, p}\left(\partial \Omega, \boldsymbol{R}^{3}\right) \rightarrow \boldsymbol{H}^{s, p}\left(\Omega, \boldsymbol{R}^{3}\right)
$$

as follows: For any $\varphi \in B^{s-1 / p, p}\left(\partial \Omega, R^{3}\right)$, the function $\mathscr{P} \varphi$ is the unique solution of the problem 


$$
\begin{cases}A v=0 & \text { in } \Omega, \\ v=\varphi & \text { on } \partial \Omega\end{cases}
$$

Next we consider the following mixed displacement-traction boundary value problem:

$$
\begin{cases}A v=\operatorname{div}(\mathbf{a} \cdot \nabla v)=f & \text { in } \Omega, \\ (\mathbf{a} \cdot \nabla v \cdot n)+v=\varphi & \text { on } \partial \Omega\end{cases}
$$

The existence and uniqueness theorem for problem (M) is also well established in the framework of Sobolev spaces of $L^{p}$ style (Theorem 3.2).

Then, using problems (D) and (M), we show that problem $(\dagger)$ can be reduced to the study of a $3 \times 3$ matrix-valued operator

$$
\boldsymbol{T}_{\alpha}:=\boldsymbol{B}_{\alpha} \mathscr{P}=\alpha \boldsymbol{I}+(1-\alpha) \boldsymbol{I},
$$

where

$$
\Pi_{\boldsymbol{\varphi}}=\left.\mathbf{a} \cdot \nabla \mathscr{P} \boldsymbol{\varphi} \cdot \boldsymbol{n}\right|_{\partial \Omega}
$$

It is known that the operator $\boldsymbol{I}$ is a $3 \times 3$ matrix-valued, classical pseudo-differential operator of first order on the boundary $\partial \Omega$.

In Section 4 we prove a regularity theorem for problem $(\dagger)$. More precisely one can construct a parametrix $\boldsymbol{S}_{\alpha}$ for the operator $\boldsymbol{T}_{\alpha}$ in the Hörmander class $L_{1,1 / 2}^{0}\left(\partial \Omega, R^{3}\right)$, and then apply a Besov-space boundedness theorem due to Bourdaud [3] to the parametrix $S_{\alpha}$ to obtain the regularity theorem for problem (†) (Theorem 4.1).

Section 5 is devoted to a uniqueness theorem for problem $(\dagger)$ (Theorem 5.1). We show that the operator

$$
\mathscr{A}_{\alpha}:=\left(A, B_{\alpha}\right): H^{s, p}\left(\Omega, R^{3}\right) \rightarrow H^{s-2, p}\left(\Omega, R^{3}\right) \times B_{(\alpha)}^{s-1-1 / p, p}\left(\partial \Omega, R^{3}\right)
$$

is injective. In the proof we make good use of Korn's inequality (Theorem 3.7) to show an inequality of Gårding type for problem $(\dagger)$.

Section 6 is devoted to an existence theorem for problem $(\dagger)$ (Theorem 6.1), which is an essential step in the proof of Main Theorem.

By the uniqueness theorem, we know that the operator $\mathscr{A}_{\alpha}$ is injective. Hence, in order to prove the surjectivity, it suffces to show that the index of the operator $\mathscr{A}_{\alpha}$ is equal to zero (Proposition 6.2).

In doing so, we replace the operator $\boldsymbol{A}$ by the operator $\boldsymbol{A}-\lambda \boldsymbol{I}$ with $\lambda \geq 0$, and consider instead of problem $(\dagger)$ the following boundary value problem:

$$
\begin{cases}(A-\lambda I) v=\operatorname{div}(\mathbf{a} \cdot \nabla v)-\lambda v=f & \text { in } \Omega, \\ \boldsymbol{B}_{\alpha} v=\alpha(\mathbf{a} \cdot \nabla v \cdot n)+(1-\alpha) v=\varphi & \text { on } \partial \Omega .\end{cases}
$$


We remark that problem $(\dagger)_{\lambda}$ coincides with problem $(\dagger)$ when $\lambda=0$.

In order to study problem $(\dagger)_{\lambda}$, we shall make use of a method essentially due to Agmon [1], just as in [13]. This is a technique of treating a spectral parameter $\lambda I$ as a second-order differential operator of an extra variable and relating the old problem to a new one with the additional variable (Propositon 6.4).

The final Section 7 is devoted to the proof of Main Theorem, Theorem 1 and Theorem 2. By Theorem 2.1, our Main Theorem follows from an application of the inverse mapping theorem. In the proof of Theorems 1 and 2, we calculate explicitly the first elasticity tensor $\AA$, and verify that condition (G) or condition (M) implies condition $(\mathrm{H})$.

I am grateful to Hiroya Ito for fruitful conversations while working on this paper. I also would like to express my hearty thanks to the referee for his careful reading of the first draft of the manuscript and many valuable suggestions.

\section{Theory of pseudo-differential operators}

In this section we present a brief description of the basic concepts and results of the $L^{p}$ theory of pseudo-differential operators which will be used in the sebsequent sections. For detailed studies of pseudo-differential operators, the reader is referred to Hörmander [7], Kumano-go [9] and Taylor [14].

1.1 Function spaces. First we recall the basic definitions and facts about the Fourier transform. If $f \in L^{1}\left(\boldsymbol{R}^{n}\right)$, we define its (direct) Fourier transform $\mathscr{F} f$ by the formula

$$
\mathscr{F} f(\xi)=\int_{\mathbf{R}^{n}} e^{-i x \cdot \xi} f(x) d x .
$$

Similarly, if $g \in L^{1}\left(R^{n}\right)$, we define its inverse Fourier transform $\mathscr{F} *^{*}$ by the formula

$$
\mathscr{F} *_{g}(x)=\frac{1}{(2 \pi)^{n}} \int_{\boldsymbol{R}^{n}} e^{i x \cdot \xi} g(\xi) d \xi
$$

We let

$\mathscr{S}\left(\boldsymbol{R}^{n}\right)=$ the space of $C^{\infty}$ functions on $\boldsymbol{R}^{n}$ rapidly decaying at infinity.

The transforms $\mathscr{F}$ and $\mathscr{F}{ }^{*} \operatorname{map} \mathscr{S}\left(\boldsymbol{R}^{n}\right)$ continuously into itself, and $\mathscr{F} \mathscr{F} *=\mathscr{F} * \mathscr{F}=\mathscr{I}$ on $\mathscr{S}\left(\boldsymbol{R}^{n}\right)$. The dual space $\mathscr{S}^{\prime}\left(\boldsymbol{R}^{n}\right)$ of $\mathscr{S}\left(\boldsymbol{R}^{n}\right)$ consists of those distributions $T \in \mathscr{D}^{\prime}\left(\boldsymbol{R}^{n}\right)$ that have continuous extensions to $\mathscr{S}\left(\boldsymbol{R}^{n}\right)$. The direct and inverse Fourier transforms can be extended to the space $\mathscr{S}^{\prime}\left(\boldsymbol{R}^{n}\right)$. Once again, the transforms $\mathscr{F}$ and $\mathscr{F} *$ map $\mathscr{S}^{\prime}\left(\boldsymbol{R}^{n}\right)$ continuously into itself, and $\mathscr{F} \mathscr{F} *=\mathscr{F} * \mathscr{F}=\mathscr{I}$ on $\mathscr{S}^{\prime}\left(\boldsymbol{R}^{n}\right)$.

If $s \in \boldsymbol{R}$, we define a linear map 


$$
J^{s}: \mathscr{S}^{\prime}\left(\boldsymbol{R}^{n}\right) \rightarrow \mathscr{S}^{\prime}\left(\boldsymbol{R}^{n}\right)
$$

by the formula

$$
J^{s} u=\mathscr{F} *\left(\left(1+|\xi|^{2}\right)^{-s / 2} \mathscr{F} u\right), \quad u \in \mathscr{S}^{\prime}\left(\boldsymbol{R}^{n}\right) .
$$

Then the map $J^{s}$ is an isomorphism of $\mathscr{S}^{\prime}\left(\boldsymbol{R}^{n}\right)$ onto itself, and its inverse is the map $J^{-s}$. The function $J^{s} u$ is called the Bessel potential of order $s$ of $u$.

The function spaces we shall treat in this paper are the following (see [2], [15]): If $s \in \boldsymbol{R}$ and $1<p<\infty$, we let

$$
H^{s, p}\left(\boldsymbol{R}^{n}\right)=\text { the image of } L^{p}\left(\boldsymbol{R}^{n}\right) \text { under the mapping } J^{s} .
$$

The space $H^{s, p}\left(\boldsymbol{R}^{n}\right)$ is called the (generalized) Sobolev space of order $s$.

We list some basic topological properties of $H^{s, p}\left(R^{n}\right)$ :

(1) The space $\mathscr{S}\left(\boldsymbol{R}^{n}\right)$ is dense in $H^{s, p}\left(\boldsymbol{R}^{n}\right)$.

(2) The space $H^{-s, p^{\prime}}\left(\boldsymbol{R}^{n}\right)$ is the dual space of $H^{s, p}\left(\boldsymbol{R}^{n}\right)$, where $p^{\prime}=p /(p-1)$ is the exponent conjugate to $p$.

(3) If $s>t$, then we have the inclusions

$$
\mathscr{S}\left(\boldsymbol{R}^{n}\right) \subset H^{s, p}\left(\boldsymbol{R}^{n}\right) \subset H^{t, p}\left(\boldsymbol{R}^{n}\right) \subset \mathscr{S}^{\prime}\left(\boldsymbol{R}^{n}\right),
$$

with continuous injections.

(4) If $s$ is a nonnegative integer, then the space $H^{s, p}\left(\boldsymbol{R}^{n}\right)$ is isomorphic to the usual Sobolev space $H^{s, p}\left(\boldsymbol{R}^{n}\right)$, that is, the space $H^{s, p}\left(\boldsymbol{R}^{n}\right)$ coincides with the space of functions $u \in L^{p}\left(\boldsymbol{R}^{n}\right)$ such that $D^{\alpha} u \in L^{p}\left(\boldsymbol{R}^{n}\right)$ for $|\alpha| \leq s$.

Next, if $1<p<\infty$, we let

$$
\begin{aligned}
& B^{1, p}\left(R^{n-1}\right)=\text { the space of functions } \varphi \in L^{p}\left(R^{n-1}\right) \text { such that } \\
& \qquad \iint_{R^{n-1} \times R^{n-1}} \frac{|\varphi(x+y)-2 \varphi(x)+\varphi(x-y)|^{p}}{|y|^{n-1+p}} d y d x<\infty .
\end{aligned}
$$

The space $B^{1, p}\left(R^{n-1}\right)$ is a Banach space with respect to the norm

$$
\begin{aligned}
|\varphi|_{1, p}= & \left(\int_{\boldsymbol{R}^{n-1}}|\varphi(x)|^{p} d x\right. \\
& \left.\quad+\iint_{\boldsymbol{R}^{n-1} \times \boldsymbol{R}^{n-1}} \frac{|\varphi(x+y)-2 \varphi(x)+\varphi(x-y)|^{p}}{|y|^{n-1+p}} d y d x\right)^{1 / p} .
\end{aligned}
$$

If $s \in \boldsymbol{R}$, we let

$$
B^{s, p}\left(R^{n-1}\right)=\text { the image of } B^{1, p}\left(R^{n-1}\right) \text { under the mapping } J^{\prime s-1},
$$

where $J^{\prime s-1}$ is the Bessel potential of order $s-1$ on $R^{n-1}$. The space $B^{s, p}\left(R^{n-1}\right)$ is called the Besov space of order $s$. 
We list some basic topological properties of $B^{s, p}\left(\boldsymbol{R}^{n-1}\right)$ :

(1) The space $\mathscr{S}\left(\boldsymbol{R}^{n-1}\right)$ is dense in $B^{s, p}\left(\boldsymbol{R}^{n-1}\right)$.

(2) The space $B^{-s, p^{\prime}}\left(\boldsymbol{R}^{n-1}\right)$ is the dual space of $B^{s, p}\left(\boldsymbol{R}^{n-1}\right)$, where $p^{\prime}=p /(p-1)$.

(3) If $s>t$, then we have the inclusions

$$
\mathscr{S}\left(\boldsymbol{R}^{n-1}\right) \subset B^{s, p}\left(R^{n-1}\right) \subset B^{t, p}\left(R^{n-1}\right) \subset \mathscr{S}^{\prime}\left(R^{n-1}\right),
$$

with continuous injections.

(4) If $s=m+\sigma$ where $m$ is a nonnegative integer and $0<\sigma<1$, then the Besov space $B^{s, p}\left(R^{n-1}\right)$ coincides with the space of functions $\varphi \in H^{m, p}\left(\boldsymbol{R}^{n-1}\right)$ such that we have, for $|\alpha|=m$,

$$
\iint_{\mathbf{R}^{n-1} \times \mathbf{R}^{n-1}} \frac{\left|D^{\alpha} \varphi(x)-D^{\alpha} \varphi(y)\right|^{p}}{|x-y|^{n-1+p \sigma}} d x d y<\infty .
$$

If $M$ is an $n$-dimensional compact $C^{\infty}$ manifold without boundary, then the spaces $H^{s, p}(M)$ are defined to be locally the spaces $H^{s, p}\left(\boldsymbol{R}^{n}\right)$ upon using local coordinate systems flattening out $M$, together with a partition of unity. The spaces $B^{s, p}(\partial \Omega)$ are defined similarly, with $H^{s, p}\left(R^{n}\right)$ replaced by $B^{s, p}\left(R^{n-1}\right)$. The norm of $B^{s, p}(\partial \Omega)$ will be denoted by $|\cdot|_{s, p}$.

Finally we state two important facts which will be used in the study of boundary value problems:

(I) The restriction map

$$
\begin{aligned}
\rho: H^{s, p}(\Omega) & \rightarrow B^{s-1 / p, p}(\partial \Omega) \\
u & \left.\mapsto u\right|_{\partial \Omega}
\end{aligned}
$$

is continuous for all $s>1 / p$, and is surjective.

(II) (Rellich) If $s>t$, then the injections

$$
\begin{gathered}
H^{s, p}(M) \rightarrow H^{t, p}(M), \\
B^{s, p}(\partial \Omega) \rightarrow B^{t, p}(\partial \Omega)
\end{gathered}
$$

are both compact (or completely continuous).

1.2 Pseudo-differential operators. Let $\Omega$ be an open subset of $\boldsymbol{R}^{n}$. If $m \in \boldsymbol{R}$ and $0 \leq \delta<\rho \leq 1$, we let

$$
\begin{aligned}
& S_{\rho, \delta}^{m}\left(\Omega \times \boldsymbol{R}^{N}\right)=\text { the set of all functions } a \in C^{\infty}\left(\Omega \times \boldsymbol{R}^{N}\right) \text { with the property } \\
& \text { that, for any compact } K \subset \Omega \text { and any multi-indices } \alpha, \beta \text {, there } \\
& \text { exists a constant } C_{K, \alpha, \beta}>0 \text { such that we have for all } x \in K \text { and } \\
& \\
& \theta \in \boldsymbol{R}^{N} \\
& \qquad\left|\partial_{\theta}^{\alpha} \partial_{x}^{\beta} a(x, \theta)\right| \leq C_{K, \alpha, \beta}(1+|\theta|)^{m-\rho|\alpha|+\delta|\beta|} .
\end{aligned}
$$

The elements of $S_{\rho, \delta}^{m}\left(\Omega \times \boldsymbol{R}^{N}\right)$ are called symbols of order $m$. We set 


$$
S^{-\infty}\left(\Omega \times \boldsymbol{R}^{N}\right)=\bigcap_{m \in R} S_{\rho, \delta}^{m}\left(\Omega \times R^{N}\right) .
$$

A symbol $a(x, \theta) \in S_{1,0}^{m}\left(\Omega \times R^{N}\right)$ is said to be classical if there exist $C^{\infty}$ functions $a_{j}(x, \theta)$, positively homogeneous of degree $m-j$ in $\theta$ for $|\theta| \geq 1$, such that, for all positive integers $k$,

$$
a-\sum_{j=0}^{k-1} a_{j} \in S_{1,0}^{m-k}\left(\Omega \times R^{N}\right)
$$

We let

$$
S_{c l}^{m}\left(\Omega \times \boldsymbol{R}^{N}\right)=\text { the set of all classical symbols of order } m .
$$

A pseudo-differential operator of order $m$ on $\Omega$ is a Fourier integral operator of the form

$$
A u(x)=\frac{1}{(2 \pi)^{n}} \iint_{\Omega \times R^{n}} e^{i(x-y) \cdot \xi} a(x, y, \xi) u(y) d y d \xi, \quad u \in C_{0}^{\infty}(\Omega),
$$

with some $a \in S_{\rho, \delta}^{m}\left(\Omega \times \Omega \times R^{n}\right)$. Here the integral is taken in the sense of oscillatory integrals.

We let

$$
L_{\rho, \delta}^{m}(\Omega)=\text { the set of all pseudo-differential operators of order } m \text { on } \Omega,
$$

and set

$$
L^{-\infty}(\Omega)=\bigcap_{m \in R} L_{\rho, \delta}^{m}(\Omega)
$$

If $A \in L_{\rho, \delta}^{m}(\Omega)$, one can choose a properly supported operator $A_{0} \in L_{\rho, \delta}^{m}(\Omega)$ such that $A-A_{0} \in L^{-\infty}(\Omega)$, and define

$$
\begin{aligned}
& \sigma(A)=\text { the equivalence class of the complete symbol of } A_{0} \text { in the } \\
& \text { factor class } S_{\rho, \delta}^{m}\left(\Omega \times R^{n}\right) / S^{-\infty}\left(\Omega \times R^{n}\right) .
\end{aligned}
$$

The equivalence class $\sigma(A)$ does not depend on the operator $A_{0}$ chosen, and is called the complete symbol of $A$.

A pseudo-differential operator $A \in L_{1,0}^{m}(\Omega)$ is said to be classical if its complete symbol $\sigma(A)$ has a representative in the class $S_{c l}^{m}\left(\Omega \times \boldsymbol{R}^{n}\right)$.

If $M$ is an $n$-dimensional paracompact $C^{\infty}$ manifold without boundary and if $m \in \boldsymbol{R}$ and $1-\rho \leq \delta<\rho \leq 1$, then we can define a class $L_{\rho, \delta}^{m}(M)$ of pseudo-differential operators of order $m$ on $M$, and transfer all the machinery of pseudo-differential operators to manifolds. We let

$L_{\rho, \delta}^{m}(M)=$ the set of all pseudo-differential operators of order $m$ on $M$.

Some results about pseudo-differential operators on $\boldsymbol{R}^{n}$ are also true for 
pseudo-differential operators on $M$. For example, we have the following three important results:

(I) The class $L_{\rho, \delta}^{m}(M)$ is stable under the operations of composition of operators and taking the transpose or adjoint of an operator.

(II) A pseudo-differential operator $A$ in the class $L_{1, \delta}^{m}(M), 0 \leq \delta<1$, extends to continuous linear operators $: H^{s, p}(M) \rightarrow H^{s-m, p}(M)$ and $A: B^{s, p}(M) \rightarrow B^{s-m, p}(M)$ for all $s \in \boldsymbol{R}$ and $1<p<\infty$ (see [3, Theorem 1]).

(III) Let $A \in L_{\rho, \delta}^{m}(M)$ with complete symbol $p(x, \xi)$. Assume that, for any compact set $K$ in each local chart $U$ and any multi-indices $\alpha, \beta$, there exist constants $C_{K, \alpha, \beta}>0, C_{K}>0$ and $\mu \in R$ such that we have, for all $x \in K$ and $|\xi| \geq C_{K}$,

$$
\begin{gathered}
\left|D_{\xi}^{\alpha} D_{x}^{\beta} p(x, \xi)\right| \leq C_{K, \alpha, \beta}|p(x, \xi)|(1+|\xi|)^{-\rho|\alpha|+\delta|\beta|}, \\
\left|p(x, \xi)^{-1}\right| \leq C_{K}(1+|\xi|)^{\mu} .
\end{gathered}
$$

Then there exists a parametrix $B$ in the class $L_{\rho, \delta}^{\mu}(M)$ for $A$ (see [7, Theorem 22.1.3]).

\section{Linear elastostatics}

In this section we study a linearization of problem $(*)$ of nonlinear elastostatics, and state our fundamental existence and unigueness theorem (Theorem 2.1) for the linearized problem $(\dagger)$ in the framework of Sobolev spaces of $L^{p}$ style.

2.1 Linearization of nonlinear elastostatics. First we linearize the nonlinear equations of elastostatics

$$
\operatorname{div} \hat{\boldsymbol{P}}(D \phi)+B=0 \quad \text { in } \Omega .
$$

The corresponding equations linearized at a configuration $\AA$ are the following (see [11, Chapter 4, Section 4.2]):

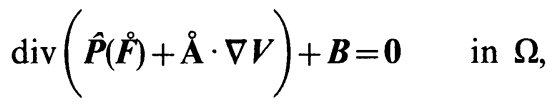

where

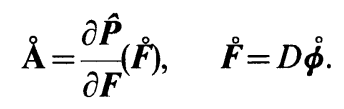

Similarly the linearization of the boundary condition

$$
\alpha \hat{\boldsymbol{P}}(D \phi) \cdot N+(1-\alpha) \phi=\tau \quad \text { on } \partial \Omega
$$

about a configuration $\phi$ is the following:

$$
\alpha(\hat{\boldsymbol{P}}(\stackrel{\circ}{\boldsymbol{F}})+\AA \cdot \boldsymbol{\nabla} \boldsymbol{V}) \cdot \boldsymbol{N}+(1-\alpha)(\stackrel{\circ}{\boldsymbol{\phi}}+\boldsymbol{V})=\tau \quad \text { on } \partial \Omega .
$$


Summing up, we obtain the following linearization of problem $(*)$ for the unknown vector function $V$ :

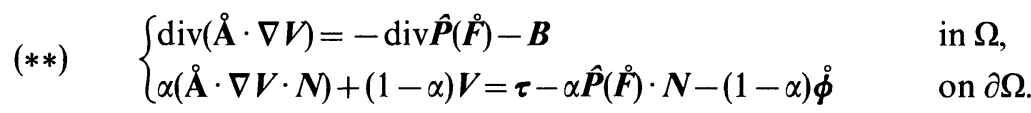

2.2 Linear elastostatics. In this subsection we consider problem $(* *)$ of linear elastostatics in the following form:

$$
\begin{cases}\operatorname{div}(\mathbf{a} \cdot \nabla v)=f & \text { in } \Omega, \\ \alpha(\mathbf{a} \cdot \nabla v \cdot n)+(1-\alpha) v=\varphi & \text { on } \partial \Omega .\end{cases}
$$

Here $\boldsymbol{a}$ is a smooth elasticity tensor and $\boldsymbol{n}$ is the outward unit normal to $\partial \Omega$.

Now we let

$$
\begin{aligned}
& A \boldsymbol{v}=\operatorname{div}(\mathbf{a} \cdot \nabla v), \\
& \boldsymbol{B}_{\alpha} \boldsymbol{v}=\alpha(\mathbf{a} \cdot \nabla \boldsymbol{v} \cdot \boldsymbol{n})+\left.(1-\alpha) v\right|_{\partial \Omega},
\end{aligned}
$$

and associate with problem $(\dagger)$ a linear operator

$$
\left(A, B_{\alpha}\right): H^{s, p}\left(\Omega, R^{3}\right) \rightarrow H^{s-2, p}\left(\Omega, R^{3}\right) \times B_{(\alpha)}^{s-1-1 / p, p}\left(\partial \Omega, R^{3}\right) .
$$

Then it is easy to verify that the operator $\left(\boldsymbol{A}, \boldsymbol{B}_{\alpha}\right)$ is continuous, for all $s>1+1 / p$.

Our fundamental result is the following existence and uniqueness theorem for problem $(\dagger)$ (cf. [8, Theorem I]):

Theorem 2.1. Let $1<p<\infty$ and $s>1 / p+1$. We assume that:

$\left(\mathrm{H}^{\prime}\right)$ The elasticity tensor a enjoys the property of symmetry

$$
\mathrm{a}_{i j l m}=\mathrm{a}_{l m i j}=\mathrm{a}_{j i l m},
$$

and is uniformly pointwise stable, that is, there is a constant $\eta>0$ such that

$$
\frac{1}{2} \boldsymbol{e} \cdot \mathbf{a} \cdot \boldsymbol{e} \geq \eta\|\boldsymbol{e}\|^{2}
$$

for all symmetric two tensors $\boldsymbol{e}$.

(A) $\alpha \neq \equiv 1$ on $\partial \Omega$.

Then the operator

$$
\left(A, B_{\alpha}\right): H^{s, p}\left(\Omega, R^{3}\right) \rightarrow H^{s-2, p}\left(\Omega, R^{3}\right) \times B_{(\alpha)}^{s-1-1 / p, p}\left(\partial \Omega, R^{3}\right)
$$

is an algebraic and topological isomorphism. 
In order to prove Theorem 2.1 , it suffices to show that the operator $\left(\boldsymbol{A}, \boldsymbol{B}_{\alpha}\right)$ is bijective. Indeed, the continuity of the inverse of $\left(\boldsymbol{A}, \boldsymbol{B}_{\alpha}\right)$ follows immediately from an application of Banach's closed graph theorem, since $\left(\boldsymbol{A}, \boldsymbol{B}_{\alpha}\right)$ is a continuous operator.

Theorem 2.1 will be proved in a series of theorems (Theorems 4.1, 5.1 and $6.1)$ in the subsequent sections.

\section{Reduction to the boundary}

In Sections 3 through 6 we study the linearized problem ( $\dagger$ ) in the framework of Sobolev spaces of $L^{p}$ style, by using the $L^{p}$ theory of pseudo-differential operators. In this section we show that problem $(\dagger)$ can be reduced to the study of a $3 \times 3$ matrix-valued pseudo-differential operator on the boundary.

3.1 Operator $T_{\alpha}$. First we consider the displacement boundary value problem

$$
\begin{cases}\operatorname{div}(\mathbf{a} \cdot \nabla v)=f & \text { in } \Omega, \\ v=\varphi & \text { on } \partial \Omega\end{cases}
$$

We let

$$
\begin{aligned}
& \boldsymbol{A v}=\operatorname{div}(\mathbf{a} \cdot \nabla v), \\
& \boldsymbol{\gamma} \boldsymbol{v}=\left.\boldsymbol{v}\right|_{\partial \Omega},
\end{aligned}
$$

and associate with problem (D) a continuous linear operator

$$
(A, \gamma): H^{s, p}\left(\Omega, R^{3}\right) \rightarrow H^{s-2, p}\left(\Omega, R^{3}\right) \times B^{s-1 / p, p}\left(\partial \Omega, R^{3}\right) .
$$

Then we have the following result (cf. [11, Chapter 6, Theorem 1.11], [8, Lemma 1.3]):

Theorem 3.1. If condition $\left(H^{\prime}\right)$ is satisfied, then the operator

$$
(A, \gamma): H^{s, p}\left(\Omega, R^{3}\right) \rightarrow H^{s-2, p}\left(\Omega, R^{3}\right) \times B^{s-1 / p, p}\left(\partial \Omega, R^{3}\right)
$$

is an algebraic and topological isomorphism, for all $s>1 / p$.

By Theorem 3.1, one can introduce a linear operator

$$
\mathscr{P}: B^{s-1 / p, p}\left(\partial \Omega, R^{3}\right) \rightarrow H^{s, p}\left(\Omega, R^{3}\right)
$$

as follows: For any $\varphi \in B^{s-1 / p, p}\left(\partial \Omega, R^{3}\right)$, the funciton $\mathscr{P} \varphi$ is the unique solution of the problem 


$$
\begin{cases}A v=0 & \text { in } \Omega \\ v=\varphi & \text { on } \partial \Omega\end{cases}
$$

The operator $\mathscr{P}$ is called the Possion operator for problem (D).

We remark that the spaces

$$
N(A, s, p)=\left\{w \in H^{s, p}\left(\Omega, R^{3}\right) ; A w=0 \quad \text { in } \Omega\right\} \quad \text { and } B^{s-1 / p, p}\left(\partial \Omega, R^{3}\right)
$$

are isomorphic in such a way that

$$
\begin{aligned}
& N(A, s, p) \stackrel{\check{r}}{\rightarrow} B^{s-1 / p, p}\left(\partial \Omega, R^{3}\right) \\
& N(A, s, p) \underset{\mathscr{P}}{\leftarrow} B^{s-1 / p, p}\left(\partial \Omega, R^{3}\right) .
\end{aligned}
$$

Next we consider the following mixed displacement-traction boundary value problem:

$$
\begin{cases}\operatorname{div}(\mathbf{a} \cdot \nabla v)=f & \text { in } \Omega, \\ (\mathbf{a} \cdot \nabla \boldsymbol{v} \cdot \boldsymbol{n})+\boldsymbol{v}=\varphi & \text { on } \partial \Omega\end{cases}
$$

We let

$$
\begin{aligned}
& A \boldsymbol{v}=\operatorname{div}(\mathbf{a} \cdot \nabla v), \\
& B v=\left.(\mathbf{a} \cdot \nabla v \cdot n)\right|_{\partial \Omega}
\end{aligned}
$$

and associate with problem (M) a continuous linear operator

$$
(A, B+\gamma): H^{s, p}\left(\Omega, R^{3}\right) \rightarrow H^{s-2, p}\left(\Omega, R^{3}\right) \times B^{s-1-1 / p, p}\left(\partial \Omega, R^{3}\right) .
$$

Then we have the following (cf. [11, Chapter 6, Theorem 1.11], [8, Lemma 1.3]):

Theorem 3.2. If condition $\left(H^{\prime}\right)$ is satisfied, then the operator

$$
(A, B+\gamma): H^{s, p}\left(\Omega, R^{3}\right) \rightarrow H^{s-2, p}\left(\Omega, R^{3}\right) \times B^{s-1-1 / p, p}\left(\partial \Omega, R^{3}\right)
$$

is an algebraic and topological isomorphism, for all $s>1+1 / p$.

Now, using problems (D) and (M), we show that problem ( $\dagger$ ) can be reduced to the study of a $3 \times 3$ matrix-valued pseudo-differential operator on the boundary.

Let $f$ be an arbitrary element of $\boldsymbol{H}^{s-2, p}\left(\boldsymbol{R}^{n}\right)$, and $\varphi$ an arbitrary element of $\boldsymbol{B}_{(\alpha)}^{s-1-1 / p, p}\left(\partial \Omega, \boldsymbol{R}^{3}\right)$ such that

$$
\varphi=\alpha \varphi_{1}+(1-\alpha) \varphi_{2}
$$

with 


$$
\varphi_{1} \in B^{s-1-1 / p, p}\left(\partial \Omega, R^{3}\right), \quad \varphi_{2} \in B^{s-1 / p, p}\left(\partial \Omega, R^{3}\right) .
$$

We assume that $\boldsymbol{u} \in \boldsymbol{H}^{s, p}\left(\Omega, \boldsymbol{R}^{3}\right)$ is a solution of the problem

$$
\begin{cases}\boldsymbol{A} \boldsymbol{u}=\boldsymbol{f} & \text { in } \Omega, \\ \boldsymbol{B}_{\alpha} \boldsymbol{u}=\alpha \boldsymbol{B} \boldsymbol{u}+(1-\alpha) \boldsymbol{u}=\varphi & \text { on } \partial \Omega .\end{cases}
$$

By Theorem 3.2, we can find an element $\boldsymbol{v} \in \boldsymbol{H}^{s, p}\left(\Omega, \boldsymbol{R}^{3}\right)$ such that

$$
\begin{cases}A v=f & \text { in } \Omega, \\ B v+\gamma v=\varphi_{1}-\varphi_{2} & \text { on } \partial \Omega .\end{cases}
$$

We let

$$
\boldsymbol{w}=\boldsymbol{u}-\boldsymbol{v} .
$$

Then it is easy to see that $\boldsymbol{w} \in \boldsymbol{H}^{s, p}\left(\Omega, \boldsymbol{R}^{3}\right)$ is a solution of the problem

$$
\begin{cases}A w=0 & \text { in } \Omega, \\ B_{\alpha} w=\varphi_{2}+(2 \alpha-1) \gamma v & \text { on } \partial \Omega .\end{cases}
$$

But the Possion operator $\mathscr{P}$ is an isomorphism of the space $B^{s-1 / p, p}\left(\partial \Omega, R^{3}\right)$ onto the space $N(A, s, p)$. Therefore we find that $\boldsymbol{w} \in \boldsymbol{H}^{s . p}\left(\Omega, R^{3}\right)$ is a solution of problem $\left(\dagger^{\prime}\right)$ if and only if $\phi \in B^{s-1 / p, p}\left(\partial \Omega, R^{3}\right)$ is a solution of the equation

$$
\boldsymbol{B}_{\alpha} \mathscr{P} \boldsymbol{\varphi}=\varphi_{2}+(2 \alpha-1) \gamma \boldsymbol{v} \quad \text { on } \partial \Omega \text {. }
$$

Here $\psi=\boldsymbol{\gamma} \boldsymbol{w}$, or equivalently, $\boldsymbol{w}=\mathscr{P} \boldsymbol{\phi}$. This is a generalization of the classical Fredholm integral equation.

Summing up, we obtain the following:

Proposition 3.3. For given $\boldsymbol{f} \in \boldsymbol{H}^{s-2, p}\left(\Omega, \boldsymbol{R}^{3}\right)$ and $\varphi \in \boldsymbol{B}_{(\alpha)}^{s-1-1 / p, p}\left(\partial \Omega, \boldsymbol{R}^{3}\right)$ with $s>1+1 / p$, there exists a solution $\boldsymbol{u} \in \boldsymbol{H}^{s, p}\left(\Omega, \boldsymbol{R}^{3}\right)$ of problem $(\dagger)$ if and only if there exists a solution $\psi \in B^{s-1 / p, p}\left(\partial \Omega, R^{3}\right)$ of equation $(\ddagger)$.

Now we let

$$
\begin{gathered}
\boldsymbol{T}_{\alpha}: \boldsymbol{C}^{\infty}\left(\partial \Omega, \boldsymbol{R}^{3}\right) \rightarrow \boldsymbol{C}^{\infty}\left(\partial \Omega, \boldsymbol{R}^{3}\right) \\
\varphi \mapsto \boldsymbol{B}_{\alpha} \mathscr{P} \varphi .
\end{gathered}
$$

Then we have

$$
\boldsymbol{T}_{\alpha}=\alpha \boldsymbol{I}+(1-\alpha) \boldsymbol{I},
$$

where 


$$
\Pi \varphi=\boldsymbol{B} \mathscr{P} \varphi=\left.\mathbf{a} \cdot \nabla \mathscr{P} \varphi \cdot \boldsymbol{n}\right|_{\partial \Omega} .
$$

It is known (see [7, Chapter XX]) that the operator $\boldsymbol{I}$ is a $3 \times 3$ matrix-valued, classical pseudo-differential operator of first order on $\partial \Omega$; hence the orepator $\boldsymbol{T}_{\alpha}$ is a $3 \times 3$ matrix-valued, classical pseudo-differential operator of first order on $\partial \Omega$.

Consequently Proposition 3.3 asserts that problem $(\dagger)$ can be reduced to the study of the system $\boldsymbol{T}_{\alpha}$ of pseudo-differential operators on the boundary $\partial \Omega$. We shall formulate this fact more precisely in terms of functional analysis.

We associate with problem $(\dagger)$ a continuous linear operator

$$
\mathscr{A}_{\alpha}=\left(A, B_{\alpha}\right): H^{s, p}\left(\Omega, R^{3}\right) \rightarrow H^{s-2, p}\left(\Omega, R^{3}\right) \times B_{(\alpha)}^{s-1-1 / p, p}\left(\partial \Omega, R^{3}\right) .
$$

Similarly we associate with equation ( $\ddagger$ ) a densely defined, closed linear operator

$$
\mathscr{T}_{\alpha}: B^{s-1 / p, p}\left(\partial \Omega, R^{3}\right) \rightarrow B^{s-1 / p, p}\left(\partial \Omega, R^{3}\right)
$$

as follows.

(a) The domain $D\left(\mathscr{T}_{\alpha}\right)$ of $\mathscr{T}_{\alpha}$ is the space

$$
D\left(\mathscr{T}_{\alpha}\right)=\left\{\varphi \in B^{s-1 / p, p}\left(\partial \Omega, R^{3}\right) ; T_{\alpha} \varphi \in B^{s-1 / p, p}\left(\partial \Omega, R^{3}\right)\right\} .
$$

(b) $\mathscr{T}_{\alpha} \varphi=T_{\alpha} \varphi, \varphi \in D\left(\mathscr{T}_{\alpha}\right)$.

Then Proposition 3.3 can be reformulated in the following form (cf. [12, Section 8.3]):

Theorem 3.4. (i) The null space $N\left(\mathscr{A}_{\alpha}\right)$ of $\mathscr{A}_{\alpha}$ has finite dimension if and only if the null space $N\left(\mathscr{T}_{\alpha}\right)$ of $\mathscr{T}_{\alpha}$ has finite dimension, and we have

$$
\operatorname{dim} N\left(\mathscr{A}_{\alpha}\right)=\operatorname{dim} N\left(\mathscr{T}_{\alpha}\right) .
$$

(ii) The range $R\left(\mathscr{A}_{\alpha}\right)$ of $\mathscr{A}_{\alpha}$ is closed if and only if the range $R\left(\mathscr{T}_{\alpha}\right)$ of $\mathscr{T}_{\alpha}$ is closed; and $R\left(\mathscr{A}_{\alpha}\right)$ has finite codimension if and only if $R\left(\mathscr{T}_{\alpha}\right)$ has finite codimiension, and we have

$$
\operatorname{codim} R\left(\mathscr{A}_{\alpha}\right)=\operatorname{codim} R\left(\mathscr{T}_{\alpha}\right) .
$$

(iii) The operator $\mathscr{A}_{\alpha}$ is a Fredholm operator if and only if the operator $\mathscr{T}_{\alpha}$ is a Fredholm operator, and we have

$$
\text { ind } \mathscr{A}_{\alpha}=\text { ind } \mathscr{T}_{\alpha} .
$$

Furthermore the next theorem states that the operator $\mathscr{A}_{\alpha}$ has regularity property if and only if the operator $\mathscr{T}_{\alpha}$ has:

Theorem 3.5. Let $1<p<\infty$ and $s>1 / p+1$. Then the following two conditions 
are equivalent:

(i) $u \in L^{p}\left(\Omega, R^{3}\right), A u \in H^{s-2, p}\left(\Omega, R^{3}\right), B_{\alpha} u \in B_{(\alpha)}^{s-1-1 / p, p}\left(\partial \Omega, R^{3}\right)$

$$
\Rightarrow \boldsymbol{u} \in \boldsymbol{H}^{s, p}\left(\Omega, \boldsymbol{R}^{3}\right) \text {. }
$$

(ii) $\varphi \in B^{-1 / p, p}\left(\partial \Omega, R^{3}\right), T_{\alpha} \varphi \in B^{s-1 / p, p}\left(\partial \Omega, R^{3}\right)$

$$
\Rightarrow \varphi \in B^{s-1 / p, p}\left(\partial \Omega, R^{3}\right) .
$$

Proof. (i) $\Rightarrow$ (ii): First, just as in [12, Proposition 8.3.2], we can prove that the boundary condition $\boldsymbol{B}_{\alpha} \boldsymbol{u}$ is defined as a function in $\boldsymbol{B}^{-1-1 / p, p}\left(\partial \Omega, R^{3}\right)$ if $\boldsymbol{u} \in L^{p}\left(\Omega, R^{3}\right)$ and $\boldsymbol{A} \boldsymbol{u} \in \boldsymbol{H}^{s-2, p}\left(\Omega, \boldsymbol{R}^{3}\right)$. Furthermore we remark that the Poisson operator $\mathscr{P}$ is an isomorphism of the space $B^{t-1 / p, p}\left(\partial \Omega, R^{3}\right)$ onto the space $N(A, t, p)=\left\{w \in H^{t, p}\left(\Omega, R^{3}\right)\right.$; $\boldsymbol{A w}=\mathbf{0}$ in $\Omega\}$ for all $\boldsymbol{t} \in \boldsymbol{R}$.

Now we assume that

$$
\varphi \in B^{-1 / p, p}\left(\partial \Omega, R^{3}\right) \quad \text { and } \quad T_{\alpha} \varphi \in B^{s-1 / p, p}\left(\partial \Omega, R^{3}\right) .
$$

Then, letteing $\boldsymbol{u}=\mathscr{P} \varphi$, we obtain that

$$
\boldsymbol{u} \in \boldsymbol{L}^{p}\left(\Omega, \boldsymbol{R}^{3}\right), \boldsymbol{A u}=0 \quad \text { and } \quad \boldsymbol{B}_{\alpha} \boldsymbol{u}=\boldsymbol{T}_{\alpha} \varphi \in B^{s-1 / p, p}\left(\partial \Omega, \boldsymbol{R}^{3}\right) .
$$

Hence it follows from condition (i) that

$$
\boldsymbol{u} \in \boldsymbol{H}^{s, p}\left(\Omega, \boldsymbol{R}^{3}\right),
$$

so that by Theorem 3.1

$$
\varphi=\gamma u \in B^{s-1 / p, p}\left(\partial \Omega, R^{3}\right) .
$$

(ii) $\Rightarrow$ (i): Conversely we assume that

$$
u \in L^{p}\left(\Omega, R^{3}\right), A u \in H^{s-2, p}\left(\Omega, R^{3}\right) \quad \text { and } \quad B_{\alpha} u \in B_{(\alpha)}^{s-1-1 / p, p}\left(\partial \Omega, R^{3}\right),
$$

where

$$
\boldsymbol{B}_{\alpha} \boldsymbol{u}=\alpha \varphi_{1}+(1-\alpha) \varphi_{2}
$$

with

$$
\varphi_{1} \in B^{s-1-1 / p, p}\left(\partial \Omega, R^{3}\right), \varphi_{2} \in B^{s-1 / p, p}\left(\partial \Omega, R^{3}\right) .
$$

Then the function $\boldsymbol{u}$ can be decomposed as follows:

$$
\boldsymbol{u}=\boldsymbol{v}+\boldsymbol{w},
$$

where $\boldsymbol{v} \in \boldsymbol{H}^{s, p}\left(\Omega, \boldsymbol{R}^{3}\right)$ is the unique solution of the problem 
(M)

$$
\begin{cases}A v=A u & \text { in } \Omega, \\ B v+\gamma v=\varphi_{1}-\varphi_{2} & \text { in } \partial \Omega,\end{cases}
$$

and so

$$
\boldsymbol{w}=\boldsymbol{u}-\boldsymbol{v} \in \boldsymbol{N}(A, 0, p) .
$$

Theorem 3.1 tells us that the function $w$ can be written as

$$
\boldsymbol{w}=\mathscr{P} \varphi, \quad \varphi=\gamma \boldsymbol{w} \in B^{-1 / p, p}\left(\partial \Omega, \boldsymbol{R}^{3}\right) .
$$

Hence we have

$$
T_{\alpha} \varphi=B_{\alpha} w=B_{\alpha} u-B_{\alpha} v=\varphi_{2}+(2 \alpha-1) \gamma v \in B^{s-1 / p, p}\left(\partial \Omega, R^{3}\right) .
$$

Thus it follows from condition (ii) that

$$
\varphi \in B^{s-1 / p, p}\left(\partial \Omega, R^{3}\right)
$$

so that again by Theorem 3.1

$$
w=\mathscr{P} \varphi \in \boldsymbol{H}^{s, p}\left(\Omega, \boldsymbol{R}^{3}\right) .
$$

This proves that

$$
u=v+w \in H^{s, p}\left(\Omega, R^{3}\right) .
$$

The proof of Theorem 3.5 is complete.

3.2 Operator $\boldsymbol{I}$. In this subsection we prove some properties of the operator $\boldsymbol{I}$ as a $3 \times 3$ matrix-valued pseudo-differential operator. In doing so, we need the following Green's formula and Korn's inequalities:

Theorem 3.6 (Green's formula). We have for all $\boldsymbol{u}, v \in C^{\infty}\left(\bar{\Omega}, R^{3}\right)$

$$
\int_{\Omega} \boldsymbol{u} \cdot \operatorname{div}(\mathbf{a} \cdot \nabla \boldsymbol{v}) d x=\int_{\partial \Omega} \boldsymbol{u}[\mathbf{a} \cdot \nabla \boldsymbol{v} \cdot \boldsymbol{n}] d a-\int_{\Omega} \nabla \boldsymbol{u} \cdot \mathbf{a} \cdot \nabla \boldsymbol{v} d x
$$

Here $d a$ is the area element on the boundary $\partial \Omega$.

By the symmetry of the tensor a, Theorem 3.6 follows from an application of the divergence theorem.

We define the strain tensor $\boldsymbol{e}=\left(e_{i j}\right)$ as

$$
e_{i j}=\frac{1}{2}\left(\frac{\partial u_{i}}{\partial x_{j}}+\frac{\partial u_{j}}{\partial x_{i}}\right)
$$

Then the next inequalities are special cases of Gårding's inequality for the elliptic 
operator $\boldsymbol{u} \mapsto \boldsymbol{e}$ (see [6, Chapitre 3, Théorèmes 3.1 et 3.3]):

Theorem 3.7 (Korn's inequalities). (i) For every non-empty open subset $\omega \subset \partial \Omega$, there exists a constant $c(\omega)>0$ such that

$$
\int_{\Omega}\|\boldsymbol{e}\|^{2} d x \geq c(\omega)\left(\int_{\Omega}\|\boldsymbol{u}\|^{2} d x+\int_{\Omega}\|\nabla \boldsymbol{u}\|^{2} d x\right)
$$

for all $\boldsymbol{u} \in \boldsymbol{H}^{1,2}\left(\Omega, \boldsymbol{R}^{3}\right)$ satisfying $\boldsymbol{u}=\mathbf{0}$ on $\omega$.

(ii) There exists a constant $c>0$ such that

$$
\int_{\Omega}\|\boldsymbol{e}\|^{2} d x+\int_{\Omega}\|\boldsymbol{u}\|^{2} d x \geq c\left(\int_{\Omega}\|\boldsymbol{u}\|^{2} d x+\int_{\Omega}\|\nabla \boldsymbol{u}\|^{2} d x\right)
$$

for all $u \in H^{1,2}\left(\Omega, R^{3}\right)$.

Now we can prove the following (cf. [8, Propositon 1.4]):

Theorem 3.8. (i) The operator $\boldsymbol{\Pi}$ is formally self-adjoint: $\Pi^{*}=\Pi$.

(ii) The operator II is strongly elliptic, that is, there exist constants $c_{1}>0$ and $c_{2}>0$ such that we have for all $\varphi \in C^{\infty}\left(\partial \Omega, C^{3}\right)$

$$
\int_{\partial \Omega} \Pi \varphi \cdot \bar{\varphi} d a \geq c_{1}|\varphi|_{1 / 2,2}^{2}-c_{2}|\varphi|_{-1 / 2,2}^{2} .
$$

(iii) The principal symbol $p_{1}\left(x^{\prime}, \xi^{\prime}\right)$ of $\boldsymbol{I}$ satifies the condition

$$
\boldsymbol{p}_{1}\left(x^{\prime}, \xi^{\prime}\right) \geq c_{0}\left|\xi^{\prime}\right| \boldsymbol{I} \quad \text { on } T^{*}(\partial \Omega),
$$

with a constant $c_{0}>0$. Here $T^{*}(\partial \Omega)$ is the cotangent bundle of $\partial \Omega$ and $\left|\xi^{\prime}\right|$ is the length of $\xi^{\prime}$ with respect to the Riemannian metric of $\partial \Omega$ induced by the natural metric of $\boldsymbol{R}^{3}$.

Proof. (i) The formal self-adjointness of $\boldsymbol{I}$ follows from the symmetry of the tensor a, by using Green's formula (3.1).

(ii) Since the tensor $\mathbf{a}$ is uniformly pointwise stable, it follows from an application of the second Korn inequality (Theorem 3.7) that, for all $\boldsymbol{u} \in \boldsymbol{H}^{1,2}\left(\Omega, C^{3}\right)$,

$$
\begin{aligned}
\int_{\Omega} \nabla \boldsymbol{u} \cdot \mathbf{a} \cdot \overline{\nabla u} d x & =\int_{\Omega} \boldsymbol{e} \cdot \mathbf{a} \cdot \overline{\boldsymbol{e}} d x \\
& \geq 2 \eta \int_{\Omega}\|\boldsymbol{e}\|^{2} d x \\
& \geq 2 \eta c\|\boldsymbol{u}\|_{1,2}^{2}-2 \eta\|\boldsymbol{u}\|_{0,2}^{2} .
\end{aligned}
$$

In particular, taking $\boldsymbol{u}=\mathscr{P} \varphi$ and using formula (3.1), we have, with $C_{1}=2 \eta c$ and 
$C_{2}=2 \eta$

$$
\int_{\partial \Omega} \Pi \varphi \cdot \bar{\varphi} d a=\int_{\Omega} \nabla \mathscr{P} \varphi \cdot \mathbf{a} \cdot \bar{\nabla} \mathscr{P} \varphi d x \geq C_{1}\|\mathscr{P} \varphi\|_{1,2}^{2}-C_{2}\|\mathscr{P} \varphi\|_{0,2}^{2}
$$

But we recall that the Poisson operator $\mathscr{P}$ is an isomorphism of $\boldsymbol{B}^{s-1 / 2,2}\left(\partial \Omega, \boldsymbol{R}^{3}\right)$ onto $N(A, s, 2)$ for all $s \in R$.

Therefore the desired inequality (3.2) follows from inequality (3.4).

(iii) It is known (see [7, Chapter XX], [9],[14]) that inequality (3.2) implies the strong ellipticity (3.3) of the operator $\boldsymbol{I}$.

\section{Regularity theorem for problem $(\dagger)$}

In this section we prove the following regularity theorem for problem $(\dagger)$ :

Theorem 4.1. Let $1<p<\infty$. If condition $\left(H^{\prime}\right)$ is satisfied, then we have, for any $s>1+1 / p$,

$$
\begin{gathered}
\boldsymbol{u} \in \boldsymbol{L}^{p}\left(\Omega, \boldsymbol{R}^{3}\right), A \boldsymbol{A} \in \boldsymbol{H}^{s-2, p}\left(\Omega, \boldsymbol{R}^{3}\right), \boldsymbol{B}_{\alpha} \boldsymbol{u} \in \boldsymbol{B}_{(\alpha)}^{s-1-1 / p, p}\left(\partial \Omega, \boldsymbol{R}^{3}\right) \\
\Rightarrow \boldsymbol{u} \in \boldsymbol{H}^{s, p}\left(\Omega, \boldsymbol{R}^{3}\right)
\end{gathered}
$$

Proof. By Theorem 3.5, we are reduced to the study of a $3 \times 3$ matrix-valued, classical pseudo-differential operator $\boldsymbol{T}_{\alpha}=\alpha \boldsymbol{I}+(1-\alpha) \boldsymbol{I}$. Hence it suffices to prove the following:

Lemma 4.2. If condition $\left(H^{\prime}\right)$ is satisfied, then we have for all $s \in \boldsymbol{R}$

$$
\varphi \in \mathscr{D}^{\prime}\left(\partial \Omega, R^{3}\right), T_{\alpha} \varphi \in B^{s, p}\left(\partial \Omega, R^{3}\right) \Rightarrow \varphi \in B^{s, p}\left(\partial \Omega, R^{3}\right) .
$$

Furthermore, for any $t<s$, there exists a constant $C_{s, t}>0$ such that

$$
|\varphi|_{s, p} \leq C_{s, t}\left(\left|T_{\alpha} \varphi\right|_{s, p}+|\varphi|_{t, p}\right)
$$

Proof. First we find that the operator $\boldsymbol{T}_{\alpha}$ is a $3 \times 3$ matrix-valued, classical pseudo-differential operator of first order on $\partial \Omega$ and its complete symbol $t\left(x^{\prime}, \xi^{\prime}\right)$ is given by the following:

$$
\begin{aligned}
\boldsymbol{t}\left(x^{\prime}, \xi^{\prime}\right)= & \alpha\left(x^{\prime}\right) \boldsymbol{p}_{1}\left(x^{\prime}, \xi^{\prime}\right)+\left[\left(1-\alpha\left(\mathrm{x}^{\prime}\right)\right) \boldsymbol{I}+\alpha\left(x^{\prime}\right) \boldsymbol{p}_{0}\left(x^{\prime}, \xi\right)\right] \\
& + \text { terms of order } \leq-1,
\end{aligned}
$$

where (cf. inequality (3.3))

$$
\boldsymbol{p}_{1}\left(x^{\prime}, \xi^{\prime}\right) \geq c_{0}\left|\xi^{\prime}\right| \boldsymbol{I} \quad \text { on } T^{*}(\partial \Omega) .
$$

Thus, just as in the proof of [13, Lemma 4.3], one can prove the following: 
Claim 4.3. If condition $\left(H^{\prime}\right)$ is satisfied, then, for each point $x^{\prime}$ of $\partial \Omega$, one can find a neighborhood $U\left(x^{\prime}\right)$ of $x^{\prime}$ such that:

For any compact $K \subset U\left(x^{\prime}\right)$ and any multi-indices $\alpha, \beta$, there exist constants $C_{K, \alpha, \beta}>0$ and $C_{K}>0$ such that we have, for all $x^{\prime} \in K$ and $\left|\xi^{\prime}\right| \geq C_{K}$,

$$
\begin{gathered}
\left\|D_{\xi^{\prime}}^{\alpha} D_{x^{\prime}}^{\beta} t\left(x^{\prime}, \xi^{\prime}\right)\right\| \leq C_{K, \alpha, \beta}\left\|\boldsymbol{t}\left(x^{\prime}, \xi^{\prime}\right)\right\|\left(1+\left|\xi^{\prime}\right|\right)^{-|\alpha|+(1 / 2)|\beta|}, \\
\left\|\boldsymbol{t}\left(x^{\prime}, \xi^{\prime}\right)^{-1}\right\| \leq C_{K^{\prime}}
\end{gathered}
$$

Therefore, applying [7, Theorem 22.1.3] to our situation, one can construct a parametrix $S_{\alpha}$ for the operator $\boldsymbol{T}_{\alpha}$ in the Hörmander class $L_{1,1 / 2}^{0}\left(\partial \Omega, \boldsymbol{R}^{3}\right)$ of $3 \times 3$ matrix-valued pseudo-differential operators on $\partial \Omega$. Lemma 4.2 follows from an application of a Besov-space boundedness theorem due to Bourdaud [3, Theorem 1].

The proof of Theorem 4.1 is complete.

\section{Uniqueness theorem for problem $(\dagger)$}

The next uniqueness theorem for problem ( $\dagger$ ) asserts that the operator $\mathscr{A}_{\alpha}$ is injective:

Theorem 5.1. Let $1<p<\infty$ and $s>1 / p+1$. Assume that conditions $(A)$ and $\left(H^{\prime}\right)$ are satisfied. If a function $\boldsymbol{v} \in \boldsymbol{H}^{s, p}\left(\Omega, \boldsymbol{R}^{3}\right)$ is a solution of the problem

$$
\begin{cases}\operatorname{div}(\mathbf{a} \cdot \nabla v)=\mathbf{0} & \text { in } \Omega, \\ \alpha(\mathbf{a} \cdot \nabla v \cdot \boldsymbol{n})+(1-\alpha) v=0 & \text { on } \partial \Omega,\end{cases}
$$

then it follows that $\mathbf{v}=\mathbf{0}$ in $\Omega$.

Proof. First, by Theorem 4.1, one may assume that

$$
v \in C^{\infty}\left(\bar{\Omega}, R^{3}\right) .
$$

Further we remark that the condition

$$
\alpha(\mathbf{a} \cdot \nabla v \cdot n)+(1-\alpha) v=0 \quad \text { on } \partial \Omega
$$

includes the condition

$$
\boldsymbol{v}=\mathbf{0} \quad \text { on the set }\{x \in \partial \Omega ; \alpha(x)=0\} .
$$

Hence it follows from an application of Green's formula (3.1) that 


$$
\begin{aligned}
0 & =\int_{\Omega} \nabla \boldsymbol{v} \cdot \mathbf{a} \cdot \nabla v d x-\int_{\partial \Omega} v[\mathbf{a} \cdot \nabla v \cdot \mathbf{n}] d a \\
& =\int_{\Omega} \boldsymbol{e} \cdot \mathbf{a} \cdot \boldsymbol{e} d x-\int_{\{\alpha \neq 0\}} v[\mathbf{a} \cdot \nabla \boldsymbol{v} \cdot \boldsymbol{n}] d a \\
& =\int_{\Omega} \boldsymbol{e} \cdot \mathbf{a} \cdot \boldsymbol{e} d x+\int_{\{\alpha \neq 0\}}\left(\frac{1-\alpha}{\alpha}\right)\|v\|^{2} d a \\
& \geq \int_{\Omega} \boldsymbol{e} \cdot \mathbf{a} \cdot \boldsymbol{e} d x,
\end{aligned}
$$

where $\boldsymbol{e}$ is the strain tensor associated with the function $\boldsymbol{v}$. But, since the elasticity tensor $\mathbf{a}$ is uniformly pointwise stable, it follows that

$$
\frac{1}{2} \boldsymbol{e} \cdot \mathbf{a} \cdot \boldsymbol{e} \geq \eta\|\boldsymbol{e}\|^{2}
$$

Hence we have

$$
0 \geq 2 \eta \int_{\Omega}\|\boldsymbol{e}\|^{2} d x
$$

and so

$$
\boldsymbol{e}=\mathbf{0} \quad \text { in } \Omega \text {. }
$$

This implies that

$$
\mathbf{0}=\alpha(\mathbf{a} \cdot \nabla \boldsymbol{v} \cdot \boldsymbol{n})+(1-\alpha) \boldsymbol{v}=\alpha(\mathbf{a} \cdot \boldsymbol{e} \cdot \boldsymbol{n})+(1-\alpha) \boldsymbol{v}=(1-\alpha) \boldsymbol{v} \quad \text { on } \partial \Omega .
$$

Thus, if we let

$$
\omega=\{x \in \partial \Omega ; \alpha(x)<1\}
$$

we find that

$$
\boldsymbol{v}=\mathbf{0} \text { on } \omega \text {. }
$$

Furthermore condition (A) tells us that the open set $\omega$ is non-empty.

Therefore we can make use of the first Korn inequality (Theorem 3.7) to obtain that

$$
\boldsymbol{v}=\mathbf{0} \quad \text { in } \Omega \text {. }
$$

Indeed, we have

$$
0 \geq 2 \eta \int_{\Omega}\|\boldsymbol{e}\|^{2} d x \geq 2 \eta c(\omega)\left(\int_{\Omega}\|\boldsymbol{v}\|^{2} d x+\int_{\Omega}\|\nabla \boldsymbol{v}\|^{2} d x\right),
$$


which proves assertion (5.1).

\section{Existence theorem for problem ( $\dagger)$}

The next existence theorem for problem ( $\dagger$ ) asserts that the operator $\mathscr{A}_{\alpha}$ is surjective:

Theorem 6.1. Let $1<p<\infty$ and $s>1 / p+1$. If conditions $(A)$ and $\left(H^{\prime}\right)$ are satisfied, then, for any $\boldsymbol{f} \in \boldsymbol{H}^{s-2, p}\left(\Omega, \boldsymbol{R}^{3}\right)$ and any $\varphi \in \boldsymbol{B}_{(\alpha)}^{s-1-1 / p, p}\left(\partial \Omega, \boldsymbol{R}^{3}\right)$, the problem

$$
\begin{cases}\operatorname{div}(\mathbf{a} \cdot \nabla v)=f & \text { in } \Omega, \\ \alpha(\mathbf{a} \cdot \nabla v \cdot \boldsymbol{n})+(1-\alpha) v=\varphi & \text { on } \partial \Omega\end{cases}
$$

has a solution $\boldsymbol{v} \in \boldsymbol{H}^{s, p}\left(\Omega, \boldsymbol{R}^{3}\right)$.

Proof. We associate with problem $(\dagger)$ a continuous linear operator

$$
\mathscr{A}_{\alpha}:=\left(A, B_{\alpha}\right): H^{s, p}\left(\Omega, R^{3}\right) \rightarrow H^{s-2, p}\left(\Omega, R^{3}\right) \times B_{(\alpha)}^{s-1-1 / p, p}\left(\partial \Omega, R^{3}\right) .
$$

Then, by Theorem 3.4, we know that

$$
\text { ind } \mathscr{A}_{\alpha}=\text { ind } \mathscr{T}_{\alpha} \text {. }
$$

But Theorem 5.1 tells us that the operator $\mathscr{A}_{\alpha}$ (or equivalently the operator $\mathscr{T}_{\alpha}$ ) is injective. Hence, in order to prove the surjectivity, it suffices to show the following:

\section{Proposition 6.2. ind $\mathscr{T}_{\alpha}=0$.}

Proof. (1) First we replace the operator $\boldsymbol{A}$ by the operator $\boldsymbol{A}-\lambda \boldsymbol{I}$ with $\lambda \geq 0$, and consider instead of problem ( $\dagger$ ) the following boundary value problem:

$$
\begin{cases}(\boldsymbol{A}-\lambda \boldsymbol{I}) \boldsymbol{u}=\boldsymbol{f} & \text { in } \Omega, \\ \boldsymbol{B}_{\alpha} \boldsymbol{u}=\alpha \boldsymbol{B} \boldsymbol{u}+(1-\alpha) \boldsymbol{u}=\boldsymbol{\varphi} & \text { on } \partial \Omega .\end{cases}
$$

We associate with problem $(\dagger)_{\lambda}$ a continuous linear operator

$$
\mathscr{A}_{\alpha}(\lambda)=\left(A-\lambda I, B_{\alpha}\right): H^{s, p}\left(\Omega, R^{3}\right) \rightarrow H^{s-2, p}\left(\Omega, R^{3}\right) \times B_{(\alpha)}^{s-1-1 / p, p}\left(\partial \Omega, R^{3}\right) .
$$

We remark that the operator $\mathscr{A}_{\alpha}(\lambda)$ coincides with the operator $\mathscr{A}_{\alpha}$ when $\lambda=0$.

We reduce the study of problem $(\dagger)_{\lambda}$ to that of a $3 \times 3$ matirx-valued pseudo-differential operator on the boundary, just as in the proof of Theorem 4.1.

We can prove that Theorem 3.1 remains valid for the operator $\boldsymbol{A}-\lambda \boldsymbol{I}$. More precisely we have the following results:

(a) The displacement boundary value problem 


$$
\begin{cases}(A-\lambda I) w=0 & \text { in } \Omega, \\ w=\varphi & \text { on } \partial \Omega\end{cases}
$$

has a unique solution $w$ in $H^{t, p}\left(\Omega, R^{3}\right)$ for any $\varphi \in B^{t-1 / p, p}\left(\partial \Omega, R^{3}\right)(t \in R)$.

(b) The Possion operator

$$
\mathscr{P}(\lambda): B^{t-1 / p, p}\left(\partial \Omega, \boldsymbol{R}^{3}\right) \rightarrow \boldsymbol{H}^{t, p}\left(\Omega, \boldsymbol{R}^{3}\right),
$$

defined by $\boldsymbol{w}=\mathscr{P}(\lambda) \varphi$, is an isomorphism of the space $B^{t-1 / p, p}\left(\partial \Omega, R^{3}\right)$ onto the space $N(A-\lambda I, t, p)=\left\{u \in H^{t, p}\left(\Omega, R^{3}\right) ;(A-\lambda I) u=0\right.$ in $\left.\Omega\right\}$ for all $t \in R$; and its inverse is the trace operator on the boundary $\partial \Omega$.

Let $T_{\alpha}(\lambda)$ be a $3 \times 3$ matrix-valued, classical pseudo-differential operator of first order on the boundary $\partial \Omega$ defined by the formula

$$
\boldsymbol{T}_{\boldsymbol{\alpha}}(\lambda)=\boldsymbol{B}_{\alpha} \mathscr{P}(\lambda)=\alpha \Pi(\lambda)+(1-\alpha) \boldsymbol{I}, \quad \lambda \geq 0,
$$

where

$$
\ddot{\Pi}(\lambda) \varphi=\boldsymbol{B} \mathscr{P}(\lambda) \varphi=\left.\mathbf{a} \cdot \nabla \mathscr{P}(\lambda) \varphi \cdot \boldsymbol{n}\right|_{\partial \Omega} .
$$

We introduce a densely defined, closed linear operator

$$
\mathscr{T}_{\alpha}(\lambda): B^{s-1 / p, p}\left(\partial \Omega, R^{3}\right) \rightarrow B^{s-1 / p, p}\left(\partial \Omega, R^{3}\right)
$$

as follows:

( $\alpha$ ) The domain $D\left(\mathscr{T}_{\alpha}(\lambda)\right)$ of $\mathscr{T}_{\alpha}(\lambda)$ is the space

$$
D\left(\mathscr{T}_{\alpha}(\lambda)\right)=\left\{\varphi \in B^{s-1 / p, p}\left(\partial \Omega, R^{3}\right) ; T_{\alpha}(\lambda) \varphi \in B^{s-1 / p, p}\left(\partial \Omega, R^{3}\right)\right\} .
$$

(B) $\mathscr{T}_{\alpha}(\lambda) \varphi=T_{\alpha}(\lambda) \varphi, \varphi \in D\left(\mathscr{T}_{\alpha}(\lambda)\right)$.

We remark that the operator $\mathscr{T}_{\alpha}(\lambda)$ coincides with the operator $\mathscr{T}_{\alpha}$ when $\lambda=0$.

Then we can obtain the following results:

(i) The null space $N\left(\mathscr{A}_{\alpha}(\lambda)\right)$ of $\mathscr{A}_{\alpha}(\lambda)$ has finite dimension if and only if the null space $N\left(\mathscr{T}_{\alpha}(\lambda)\right)$ of $\mathscr{T}_{\alpha}(\lambda)$ has finite demension, and we have

$$
\operatorname{dim} N\left(\mathscr{A}_{\alpha}(\lambda)\right)=\operatorname{dim} N\left(\mathscr{T}_{\alpha}(\lambda)\right)
$$

(ii) The range $R\left(\mathscr{A}_{\alpha}(\lambda)\right)$ of $\mathscr{A}_{\alpha}(\lambda)$ is closed if and only if the range $R\left(\mathscr{T}_{\alpha}(\lambda)\right)$ of $\mathscr{T}_{\alpha}(\lambda)$ is closed; and $R\left(\mathscr{A}_{\alpha}(\lambda)\right)$ has finite codimension if and only if $R\left(\mathscr{T}_{\alpha}(\lambda)\right.$ ) has finite codimension, and we have

$$
\operatorname{codim} R\left(\mathscr{A}_{\alpha}(\lambda)\right)=\operatorname{codim} R\left(\mathscr{T}_{\alpha}(\lambda)\right) .
$$

(iii) The operator $\mathscr{A}_{\alpha}(\lambda)$ is a Fredholm operator if and only if the operator $\mathscr{T}_{\alpha}(\lambda)$ is a Fredholm operator, and we have

$$
\text { ind } \mathscr{A}_{\alpha}(\lambda)=\operatorname{ind} \mathscr{T}_{\alpha}(\lambda) \text {. }
$$


(2) In order to study problem $(\dagger)_{\lambda}$, we shall make use of a method essentially due to Agmon [1] (see [12, Section 8.4], [10]).

We introduce an auxiliary variable $y$ of the unit circle

$$
S=R / 2 \pi Z
$$

and replace the parameter $-\lambda I$ by the second-order differential operator

$$
\frac{\partial^{2}}{\partial y^{2}} I
$$

That is, we replace the operator $A-\lambda I$ by the operator

$$
\tilde{\Lambda}=A+\frac{\partial^{2}}{\partial y^{2}} I
$$

and consider instead of problem $(\dagger)_{\lambda}$ the following boundary value problem:

$$
\begin{cases}\tilde{\boldsymbol{\Lambda}} \tilde{\boldsymbol{u}}=\left(\boldsymbol{A}+\frac{\partial^{2}}{\partial y^{2}} I\right) \tilde{\boldsymbol{u}}=\tilde{f} & \text { in } \Omega \times S, \\ \boldsymbol{B}_{\alpha} \tilde{\boldsymbol{u}}=\alpha \boldsymbol{B} \tilde{\boldsymbol{u}}+(1-\alpha) \tilde{\boldsymbol{u}}=\tilde{\varphi} & \text { on } \partial \Omega \times S .\end{cases}
$$

Then we have the following results:

(ã) The displacement boundary value problem

$$
\begin{cases}\tilde{\boldsymbol{\Lambda}} \tilde{\boldsymbol{w}}=\mathbf{0} & \text { in } \Omega \times S, \\ \tilde{\boldsymbol{w}}=\tilde{\varphi} & \text { on } \partial \Omega \times S\end{cases}
$$

has a unique solution $\tilde{\boldsymbol{w}}$ in $\boldsymbol{H}^{t, p}\left(\Omega \times S, \boldsymbol{R}^{3}\right)$ for any $\tilde{\varphi} \in \boldsymbol{B}^{t-1 / p, p}\left(\partial \Omega \times S, \boldsymbol{R}^{3}\right)(t \in \boldsymbol{R})$.

(b) The Poission operator

$$
\tilde{\mathscr{P}}: \boldsymbol{B}^{t-1 / p, p}\left(\partial \Omega \times S, \boldsymbol{R}^{3}\right) \rightarrow \boldsymbol{H}^{t, p}\left(\Omega \times S, \boldsymbol{R}^{3}\right),
$$

defined by $\tilde{\boldsymbol{w}}=\tilde{\mathscr{P}} \tilde{\boldsymbol{\varphi}}$, is an isomorphism of the space $\boldsymbol{B}^{t-1 / p, p}\left(\partial \Omega \times S, \boldsymbol{R}^{3}\right)$ onto the space $N(\tilde{\boldsymbol{\Lambda}}, t, p)=\left\{\tilde{\boldsymbol{u}} \in \boldsymbol{H}^{t, p}\left(\Omega \times S, \boldsymbol{R}^{3}\right) ; \tilde{\boldsymbol{\Lambda}} \tilde{\boldsymbol{u}}=\mathbf{0}\right.$ in $\left.\Omega \times S\right\}$ for all $t \in \boldsymbol{R}$; and its inverse is the trace operator on the boundary $\partial \Omega \times S$.

We let

$$
\begin{gathered}
\tilde{\boldsymbol{T}}_{\alpha}: C^{\infty}\left(\partial \Omega \times S, \boldsymbol{R}^{3}\right) \rightarrow C^{\infty}\left(\partial \Omega \times S, \boldsymbol{R}^{3}\right) \\
\tilde{\varphi} \mapsto \boldsymbol{B}_{\alpha} \tilde{\mathcal{P}} \tilde{\varphi} .
\end{gathered}
$$

Then the operator $\tilde{T}_{\alpha}$ can be decomposed as follows:

$$
\tilde{\boldsymbol{T}}_{\alpha}=\alpha \tilde{\boldsymbol{I}}+(1-\alpha) \boldsymbol{I},
$$

where

$$
\tilde{\boldsymbol{M}} \tilde{\varphi}=\boldsymbol{B} \tilde{\mathscr{P}} \tilde{\varphi}=\left.\mathbf{a} \cdot \nabla \tilde{\mathscr{P}} \tilde{\varphi} \cdot \boldsymbol{n}\right|_{\hat{\partial} \Omega \times S}
$$


The operator $\tilde{\boldsymbol{I}}$ is a $3 \times 3$ matrix-valued, classical pseudo-differential operator of first order on $\partial \Omega \times S$, and its complete symbol $\tilde{\boldsymbol{p}}_{1}\left(x^{\prime}, \xi^{\prime}, y, \eta\right)$ is given by the following:

$$
\tilde{\boldsymbol{p}}_{1}\left(x^{\prime}, \xi^{\prime}, y, \eta\right)+\tilde{\boldsymbol{p}}_{0}\left(x^{\prime}, \xi^{\prime}, y, \eta\right)+\text { terms of order } \leq-1,
$$

where (cf. inequality (4.2))

$$
\tilde{\boldsymbol{p}}_{1}\left(x^{\prime}, \xi^{\prime}, y, \eta\right) \geq \tilde{c}_{0} \sqrt{\left|\xi^{\prime}\right|^{2}+\eta^{2}} \boldsymbol{I} \quad \text { on } \quad T^{*}(\partial \Omega \times S) .
$$

Thus we find that the operator $\tilde{\boldsymbol{T}}_{\alpha}=\alpha \tilde{\boldsymbol{I}}+(1-\alpha) \boldsymbol{I}$ is a $3 \times 3$ matrix-valued, classical pseudo-differential operator of first order on $\partial \Omega \times S$ and its complete symbol $z^{\prime}\left(x^{\prime}, \xi^{\prime}, y, \eta\right)$ is given by the following (cf. formula (4.1)):

$$
\begin{aligned}
\boldsymbol{z}\left(x^{\prime}, \xi^{\prime}, y, \eta\right)= & \alpha\left(x^{\prime}\right) \tilde{\boldsymbol{p}}_{1}\left(x^{\prime}, \xi^{\prime}, y, \eta\right)+\left[\left(1-\alpha\left(x^{\prime}\right)\right) \boldsymbol{I}+\alpha\left(x^{\prime}\right) \tilde{\boldsymbol{p}}_{0}\left(x^{\prime}, \xi^{\prime}, y, \eta\right)\right] \\
& + \text { terms of order } \leq-1 .
\end{aligned}
$$

Then, by virtue of formulas (6.2) and (6.1), it is easy to verify that the operator $\tilde{\boldsymbol{T}}_{\alpha}$ satisfies all the conditions of a matrix-valued version of [7, Theorem 22.1.3] with $\mu=0, \rho=1$ and $\delta=1 / 2$, just as in Lemma 4.2. Hence there exists a parametrix $\tilde{\boldsymbol{S}}_{\alpha}$ in the Hörmander class $\boldsymbol{L}_{1,1 / 2}^{0}\left(\partial \Omega \times S, \boldsymbol{R}^{3}\right)$ for the operator $\tilde{\boldsymbol{T}}_{\alpha}$.

Therefore we obtain the following result, analogous to Lemma 4.2:

Lemma 6.3. If condition $\left(H^{\prime}\right)$ is satisfied, then we have for all $s \in \boldsymbol{R}$

$$
\tilde{\boldsymbol{\varphi}} \in \mathscr{D}^{\prime}(\partial \Omega \times S), \quad \tilde{\boldsymbol{T}}_{\alpha} \tilde{\boldsymbol{\varphi}} \in \boldsymbol{B}^{s, p}\left(\partial \Omega \times S, \boldsymbol{R}^{3}\right) \Rightarrow \tilde{\boldsymbol{\varphi}} \in \boldsymbol{B}^{s, p}\left(\partial \Omega \times S, \boldsymbol{R}^{3}\right) .
$$

Furthermore, for any $t<s$, there exists a constant $\tilde{C}_{s, t}>0$ such that

$$
|\tilde{\varphi}|_{s, p} \leq \tilde{C}_{s, t}\left(\left|\tilde{\boldsymbol{T}}_{\alpha} \tilde{\boldsymbol{\varphi}}\right|_{s, p}+|\tilde{\varphi}|_{t, p}\right) \text {. }
$$

We introduce a densely defined, closed linear operator

$$
\tilde{\mathscr{T}}_{\alpha}: \boldsymbol{B}^{s-1 / p, p}\left(\partial \Omega \times S, \boldsymbol{R}^{3}\right) \rightarrow \boldsymbol{B}^{s-1 / p, p}\left(\partial \Omega \times S, \boldsymbol{R}^{3}\right)
$$

as follows:

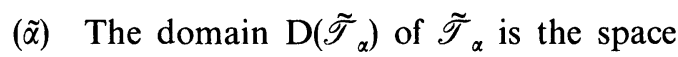

$$
D\left(\tilde{\mathscr{T}}_{\alpha}\right)=\left\{\tilde{\boldsymbol{\varphi}} \in B^{s-1 / p, p}\left(\partial \Omega \times S, \boldsymbol{R}^{3}\right) ; \tilde{\boldsymbol{T}}_{\alpha} \tilde{\boldsymbol{\varphi}} \in \boldsymbol{B}^{s-1 / p, p}\left(\partial \Omega \times S, \boldsymbol{R}^{3}\right)\right\} .
$$

( $\widetilde{\beta}) \quad \tilde{\mathscr{T}}_{\alpha} \tilde{\boldsymbol{\varphi}}=\tilde{\boldsymbol{T}}_{\alpha} \tilde{\boldsymbol{\varphi}}, \tilde{\boldsymbol{\varphi}} \in D\left(\tilde{\mathscr{T}}_{\alpha}\right)$.

Then we have the following fundamental relationship between the operators $\tilde{\mathscr{T}}_{\alpha}$ and $\mathscr{T}_{\alpha}(\lambda)$, just as in [13, Proposition 6.2]:

Proposition 6.4. If ind $\tilde{\mathscr{T}}_{\alpha}$ is finite, then there exists a finite subset $K$ of $\boldsymbol{Z}$ such that the operator $\mathscr{T}_{a}\left(\lambda^{\prime}\right)$ is bijective for all $\lambda^{\prime}=l^{2}$ satisfying $l \in Z \backslash K$. 
(3) We show that if condition $\left(H^{\prime}\right)$ is satisfied, then we have

$$
\text { ind } \tilde{\mathscr{T}}_{\alpha}=\operatorname{dim} N\left(\tilde{\mathscr{T}}_{\alpha}\right)-\operatorname{codim} R\left(\tilde{\mathscr{T}}_{\alpha}\right)<\infty \text {. }
$$

Now estimate (6.3) gives that

$$
|\tilde{\varphi}|_{s-1 / p, p} \leq \tilde{C}_{t}\left(\left|\tilde{\mathscr{T}}_{\alpha} \tilde{\varphi}\right|_{s-1 / p, p}+|\tilde{\varphi}|_{t, p}\right), \quad \tilde{\varphi} \in D\left(\tilde{\mathscr{T}}_{\alpha}\right),
$$

where $t<s-1 / p$. But it follows from an application of Rellich's theorem that the injection $\boldsymbol{B}^{s-1 / p, p}\left(\partial \Omega \times S, \boldsymbol{R}^{3}\right) \rightarrow \boldsymbol{B}^{t, p}\left(\partial \Omega \times S, \boldsymbol{R}^{3}\right)$ is compact for $t<s-1 / p$. Thus, applying the well-known Peetre lemma (see [12, Theorem 3.7.6]) to our situation, we obtain that the range $R\left(\tilde{\mathscr{T}}_{\alpha}\right)$ is closed in $B^{s-1 / p, p}\left(\partial \Omega \times S, R^{3}\right)$ and

$$
\operatorname{dim} N\left(\tilde{\mathscr{T}}_{\alpha}\right)<\infty .
$$

Similarly, by formula (6.2), one can easily verify that the adjoint $\tilde{\boldsymbol{T}}^{*}$ also satisfies all the conditions of a matrix-valued version of [7, Theorem 22.1.3] with $\mu=0$, $\rho=1$ and $\delta=1 / 2$. This implies that estimate (6.5) remains valid for the adjoint operator $\tilde{\mathscr{T}}_{\alpha}^{*}$ of $\tilde{\mathscr{T}}_{\alpha}$ :

$$
|\tilde{\psi}|_{-s+1 / p, p} \leq \tilde{C}_{\tau}\left(\left|\tilde{\mathscr{T}}_{\alpha}^{*} \tilde{\boldsymbol{\psi}}\right|_{-s+1 / p, p^{\prime}}+|\tilde{\boldsymbol{\psi}}|_{\tau, p^{\prime}}\right), \quad \tilde{\boldsymbol{\psi}} \in D\left(\tilde{\mathscr{T}}_{\alpha}^{*}\right),
$$

where $\tau<-s+1 / p$ and $p^{\prime}=p /(p-1)$, the exponent conjugate to $p$. Hence we have by the closed range theorem and Peetre's lemma

$$
\operatorname{codim} R\left(\tilde{\mathscr{T}}_{\alpha}\right)=\operatorname{dim} N\left(\tilde{\mathscr{T}}_{\alpha}^{*}\right)<\infty,
$$

since the injection $B^{-s-1 / p, p^{\prime}}\left(\partial \Omega \times S, R^{3}\right) \rightarrow B^{\tau, p^{\prime}}\left(\partial \Omega \times S, R^{3}\right)$ is compact for $\tau<$ $-s+1 / p$.

Therefore assertion (6.4) follows from assertions (6.6) and (6.7).

(4) By assertion (6.4), we can apply Proposition 6.4 to obtain that the operator $\mathscr{T}_{\alpha}\left(l^{2}\right): \boldsymbol{B}^{s-1 / p, p}\left(\partial \Omega, \boldsymbol{R}^{3}\right) \rightarrow \boldsymbol{B}^{s-1 / p, p}\left(\partial \Omega, \boldsymbol{R}^{3}\right)$ is bijective if $l \in Z \backslash K$ for some finite subset $K$ of $Z$. In particular we have

$$
\text { ind } \mathscr{T}_{\alpha}\left(\lambda_{0}\right)=0 \quad \text { if } \quad \lambda_{0}=l^{2}, \quad l \in Z \backslash K \text {. }
$$

But one can find a $3 \times 3$ matrix-valued, classical pseudo-differential operator $K\left(\lambda_{0}\right)$ of order -1 on $\partial \Omega$ such that (taking $\lambda=0$ )

$$
T_{\alpha}=T_{\alpha}\left(\lambda_{0}\right)+K\left(\lambda_{0}\right)
$$

Furthermore Rellich's theorem tells us that the operator

$$
K\left(\lambda_{0}\right): B^{s-1 / p, p}\left(\partial \Omega, R^{3}\right) \rightarrow B^{s-1 / p, p}\left(\partial \Omega, R^{3}\right)
$$

is compact. Hence we have

$$
\text { ind } \mathscr{T}_{\alpha}=\text { ind } \mathscr{T}_{\alpha}\left(\lambda_{0}\right) \text {. }
$$


Therefore Proposition 6.2 follows by combining assertions (6.8) and (6.9).

The proof of Theorem 6.1 is now complete.

\section{Proof of theorems}

This final seciton is devoted to the proof of Main Theorem, Theorem 1 and Theorem 2. Main Theorem follows from an application of the inverse mapping theorem. In the proof of Theorems 1 and 2, we calculate explicitly the first elasticity tensor $\AA$, and verify condition $(\mathrm{H})$ in Main Theorem.

7.1 Proof of main theorem. We recall that the linearization of problem $(*)$ is problem $(* *)$ or problem $(\dagger)$ as is shown in Section 2. But Theorem 2.1 (the existence and uniqueness theorem for problem $(\dagger))$ tells us that:

The Fréchet derivative $F^{\prime}(\grave{\phi})$ of the map $F$ at $\stackrel{\phi}{=}=I_{\Omega}$ is an algebraic and topological isomorphism of $\boldsymbol{H}^{s, p}\left(\Omega, \boldsymbol{R}^{3}\right)$ onto $\boldsymbol{H}^{s-2, p}\left(\Omega, \boldsymbol{R}^{3}\right) \times \boldsymbol{E}_{(\alpha)}^{s-1-1 / p, p}\left(\partial \Omega, \boldsymbol{R}^{3}\right)$.

Therefore Main Theorem follows immediately from an application of the inverse mapping theorem (see [11, Chapter 4, Theorem 1.2]).

7.2 Proof of theorem 1. The stored energy function for the Hencky-Nadai elasto-plastic material has the form

$$
W(X, F)=\frac{3}{4} \int_{0}^{T(\boldsymbol{F})} g(\xi) d \xi+\frac{K}{2}\left(\sum_{k=1}^{3} F_{k k}-3\right)^{2} .
$$

We have only to verify condition $(\mathrm{H})$. First it follows that the first Piola-Kirchhoff stress tensor is given by the formula

$$
\hat{P}_{i j}(X, F)=\left(K-\frac{2}{3} g(\Gamma(\boldsymbol{F}))\right)\left(\sum_{k=1}^{3} F_{k k}-3\right) \delta_{i j}+g(\Gamma(\boldsymbol{F}))\left(F_{i j}+F_{j i}-2 \delta_{i j}\right)
$$

and the first elasticity tensor is given by the formula

$$
\begin{aligned}
\mathrm{A}_{i j l m}(X, \boldsymbol{F})= & g(\Gamma(\boldsymbol{F}))\left(\delta_{i l} \delta_{j m}+\delta_{i m} \delta_{j l}\right)+\left(K-\frac{2}{3} g(\Gamma(\boldsymbol{F}))\right) \delta_{i j} \delta_{l m} \\
& +\frac{16}{3} g^{\prime}(\Gamma(\boldsymbol{F})) \tilde{\varepsilon}_{i j}(\boldsymbol{F}) \tilde{\varepsilon}_{l m}(\boldsymbol{F}),
\end{aligned}
$$

where 


$$
\tilde{\varepsilon}_{i j}(\boldsymbol{F})=\frac{1}{2}\left(F_{i j}+F_{j i}\right)-\frac{1}{3}\left(\sum_{k=1}^{3} F_{k k} F_{k k}\right) \delta_{i j}
$$

Thus we find that the elasticity tensor $\AA$ evaluated at $\stackrel{\phi}{\phi}=\boldsymbol{I}_{\Omega}$ is equal to the following:

$$
\AA_{i j l m}(X)=g(0)\left(\delta_{i l} \delta_{j m}+\delta_{i m} \delta_{j l}\right)+\left(K-\frac{2}{3} g(0)\right) \delta_{i j} \delta_{l m}
$$

But it is easy to verify (see [11, Chapter 4, Proposition 3.13]) that the elasticity tensor $\AA$ is uniformly pointwise stable if and only if $g(0)>0$ and $K>0$.

Therefore we have proved that condition $(\mathrm{G})$ implies condition $(\mathrm{H})$.

7.3 Proof of theorem 2. The stored energy function for the St.VenantKirchhoff isotropic material has the form

$$
W(X, F)=\frac{\lambda(X)}{8}\left(\sum_{k=1}^{3} C_{k k}(\boldsymbol{F})-3\right)^{2}+\frac{\mu(X)}{4} \sum_{i, j=1}^{3}\left(C_{i j}(F)-\delta_{i j}\right)^{2}
$$

We verify condition $(\mathrm{H})$. First it follows that the second Piola-Kirchhoff stress tensor is given by

$$
\hat{S}_{i j}(X, C)=2 \frac{\partial W}{\partial C_{i j}}(X, C)=\left[\frac{\lambda(X)}{2}\left(\sum_{k=1}^{3} C_{k k}-3\right)-\mu(X)\right] \delta_{i j}+\mu(X) C_{i j},
$$

and the second elasticity tensor is given by

$$
C_{i j l m}(X, C)=\frac{\partial \hat{S}_{i j}}{\partial C_{l m}}(X, C)=\frac{\lambda(X)}{2} \delta_{i j} \delta_{l m}+\frac{\mu(X)}{2}\left(\delta_{i l} \delta_{j m}+\delta_{i m} \delta_{j l}\right)
$$

Then we know (see [11, Chapter 3, Proposition 4.5]) that the first elasticity tensor is given by the following formula:

$$
\begin{aligned}
\mathrm{A}_{i j l m}(X, \boldsymbol{F})= & 2 \sum_{a, b=1}^{3} C_{a j b m}(X, \boldsymbol{F}) F_{i a} F_{l b}+\hat{S}_{j m}(X, \boldsymbol{F}) \delta_{i l} \\
= & \sum_{a, b=1}^{3}\left(\lambda(X) \delta_{a j} \delta_{b m}+\mu(X)\left(\delta_{a b} \delta_{j m}+\delta_{a m} \delta_{b j}\right)\right) F_{i a} F_{l b} \\
& +\left(\left[\frac{\lambda(X)}{2}\left(\sum_{k=1}^{3} C_{k k}(\boldsymbol{F})-3\right)-\mu(X)\right] \delta_{j m}+\mu(X) C_{j m}(\boldsymbol{F})\right) \delta_{i l} .
\end{aligned}
$$

Thus it follows that the elasticity tensor $\AA$ evaluated at $\stackrel{\phi}{=} \boldsymbol{I}_{\boldsymbol{\Omega}}$ is equal to the following:

$$
\AA_{i j l m}(X)=\mu(X)\left(\delta_{i l} \delta_{j m}+\delta_{i m} \delta_{j l}\right)+\lambda(X) \delta_{i j} \delta_{l m} .
$$


But it is easy to verify (see [11, Chapter 4, Proposition 3.13]) that if condition (M) is satisfied, then the elasticity tensor $\AA$ is uniformly pointwise stable. This proves that condition $(\mathrm{M})$ implies condition $(\mathrm{H})$.

\section{References}

[1] S. Agmon: Lectures on elliptic boundary value problems, Van Nostrand, Princeton, 1965.

[2] J. Bergh and J. Löfstörm, Interpolation spaces, an introduction, Springer-Verlag, Berlin New York Heidelberg, 1976.

[3] G. Bourdaud: $L^{p}$-estimates for certain non-regular pseudo-differential operators, Comm. Partial Differential Equations 7 (1982), 1023-1033.

[4] P.G. Ciarlet: Mathematical elasticity, vol.I, Studies in mathematics and its applications, no. 20, North-Holland, Amsterdam New York Oxford Tokyo, 1988.

[5] G. Dinca: Sur la monotonie d'après Minty-Browder de Topérateur de la théorie de plasticité, C.R. Acad. Sci. Paris 269 (1969), 535-538.

[6] G. Duvaut et J.-L. Lions: Les inéquations en mécanique et en physique, Dunod, Paris, 1972.

[7] L. Hörmander, The analysis of linear partial differential operators, vol. III, Springer-Verlag, New York Berlin Heidelberg Tokyo, 1983.

[8] H. Ito: On certain mixed-type boundary-value problems of elastostatics, Tsukuba J. Math. 14 (1990), 133-153.

[9] H. Kumano-go: Pseudo-differential operators, MIT Press, Cambridge, Massachusetts, 1981.

[10] J.-L. Lions et E. Magenes: Problèmes aux limites non-homogènes et applications, vols. 1, 2, Dunod, Paris, 1968; English translation, Springer-Verlag, Berlin New York Heidelberg, 1972.

[11] J.E. Marsden and T.J.R.Hughes: Mathematical foundations of elasticity, Prentice-Hall, Inc., Englewood Cliffs, New Jersey, 1983.

[12] K. Taira: Diffusion processes and partial differential equations, Academic Press, San Diego New York London Tokyo, 1988.

[13] K. Taira: Boundary value problems and Markov processes, Lecture Notes in Math., no.1499, Springer-Verlag, Berlin Heidelberg New York Tokyo, 1991.

[14] M. Taylor: Pseudodifferential operators, Princeton Univ. Press, Princeton, 1981.

[15] H. Triebel: Interpolation theory, function spaces, differential operators, North-Holland, Amsterdam, 1978.

[16] T. Valent: Boundary value problems of finite elasticity, Springer tracts in natural philosophy, no.31, Springer-Verlag, New York Berlin Heidelberg Tokyo, 1988.

Institute of Mathematics

University of Tsukuba

Tsukuba 305, Japan

Current address:

Department of Mathematics

Hiroshima University

Higashi-Hiroshima 739, Japan 
